\title{
Incidence of primary graft dysfunction is higher according to the new ISHLT 2016 guidelines and correlates with clinical and molecular risk factors
}

\author{
Daoud Daoud ${ }^{1}$, Lourdes Chacon Alberty ${ }^{2}$, Qi Wei ${ }^{1}$, Camila Hochman Mendez ${ }^{2}$, \\ Muhammad Hassan Masood Virk ${ }^{3}$, Jonathan Mase ${ }^{2}$, Peter Jindra ${ }^{1}$, Matthew Cusick ${ }^{1}$, Hyewon Choi ${ }^{1}$, \\ Natalie Debolske ${ }^{1}$, Luiz C. Sampaio ${ }^{4}$, Doris A. Taylor ${ }^{5}$, Gabriel Loor ${ }^{1}$ \\ ${ }^{1}$ Michael E DeBakey Department of Surgery, Division of Cardiopulmonary Transplantation and Mechanical Circulatory Support, Baylor College \\ of Medicine, Houston, TX, USA; ${ }^{2}$ Department of Regenerative Medicine Research, Texas Heart Institute, Houston, TX, USA; ${ }^{3}$ Center for \\ Antimicrobial Resistance and Microbial Genomics (CARMiG), Department of Internal Medicine, Division of Infectious Diseases, University \\ of Texas Health Science Centre at Houston, Houston, TX, USA; ${ }^{4}$ Department of Advanced Cardiopulmonary Therapies and Transplantation, \\ University of Texas Health Science Center at Houston, Houston, TX, USA; ${ }^{5}$ RegenMedix Consulting LLC, Montgomery, AL, USA \\ Contributions: (I) Conception and design: L Chacon Alberty, DA Taylor, G Loor; (II) Administrative support: C Hochman Mendez, LC Sampaio, \\ DA Taylor, G Loor; (III) Provision of study materials or patients: P Jindra, DA Taylor, G Loor; (IV) Collection and assembly of data: D Daoud, L \\ Chacon Alberty, MHM Virk, J Mase, M Cusick, H Choi, N Debolske; (V) Data analysis and interpretation: D Daoud, L Chacon Alberty, Q Wei, \\ MHM Virk, J Mase, P Jindra, DA Taylor, G Loor; (VI) Manuscript writing: All authors; (VII) Final approval of manuscript: All authors. \\ Correspondence to: Gabriel Loor. Michael E DeBakey Department of Surgery, Division of Cardiopulmonary Transplantation and Mechanical \\ Circulatory Support, Baylor College of Medicine, Houston, TX, USA. Email: Gabriel.loor@bcm.edu.
}

Background: Primary graft dysfunction (PGD) is the most important determinant of morbidity and mortality after lung transplantation, but its definition has evolved over the past decade. The implications of this refinement in clinical definition have not been evaluated. In this single-center study, we compared PGD incidence, risk factors, and outcomes using the 2005 and the updated-2016 International Society of Heart and Lung Transplantation guidelines for PGD grading in lung transplant patients.

Methods: In this retrospective study, we extracted data from the medical records of 127 patients who underwent lung transplantation between 1/1/2016-12/31/2018. PGD was defined as PGD3 present at 48 and/or 72 hours post-reperfusion. We used the 2005 and the updated 2016 guidelines to assess clinical risk factors, outcomes, and baseline biomarkers for PGD.

Results: On the basis of the 2016 and 2005 guidelines, we identified PGD in 37\% and 26\% of patients, respectively. PGD was significantly associated with extracorporeal life support, large body mass index, and restrictive lung disease using the 2016 but not the 2005 guidelines. Based on the 2016 guidelines, pretransplant levels of several biomarkers were associated with PGD; using the 2005 guidelines, only increased interleukin-2 levels were significantly associated with PGD. No preoperative biomarkers were associated with PGD using either guidelines after adjusting for clinical variables. Postoperative morbidity and 1-year mortality were similar regardless of guidelines used.

Conclusions: Our findings suggest that refinements in the PGD scoring system have improved the detection of graft injury and associated risk factors without changing its ability to predict postoperative morbidity and mortality.

Keywords: Lung transplantation; risk factors; primary graft dysfunction (PGD); cytokines; lung disease

Submitted Jan 05, 2021. Accepted for publication Apr 14, 2021.

doi: $10.21037 /$ jtd-20-3564

View this article at: http://dx.doi.org/10.21037/jtd-20-3564

(c) Journal of Thoracic Disease. All rights reserved. 


\section{Introduction}

Primary graft dysfunction (PGD) after lung transplantation is a primary determinant of morbidity and mortality. However, the definition of PGD has evolved over the past decade, and the analysis of the effects of these refinements on the ability to detect lung injury, risk factors, or clinical outcomes has been limited. The most widely used PGD scoring system has been the 2005 International Society of Heart and Lung Transplantation (ISHLT) guidelines based on the degree of hypoxia and pulmonary edema on chest radiograph (CXR), which have aimed to standardize PGD diagnosis. PGD is often graded on a scale of $0-3$; PGD3 occurs when the ratio between partial pressure of oxygen and fraction of inspired oxygen $\left(\mathrm{PaO}_{2}: \mathrm{FiO}_{2}\right.$ ratio $)$ is $<200$ and edema is present on CXR, according to the 2005 guidelines (1).

Over a decade's worth of information on risk factors and clinical significance of PGD is based on the use of these guidelines (2-8). A 2016 guidelines update (9) clarified the grading of extubated patients by advocating for using the patient's estimated $\mathrm{FiO}_{2}$ in the $\mathrm{PaO}_{2}: \mathrm{FiO}_{2}$ ratio rather than CXR findings alone, regardless of the mode of non-invasive ventilation. Additional clarifications included the use of a saturation scale for patients who may not have an arterial monitoring line, the grading of patients on extracorporeal membrane oxygenation (ECMO), and improved clarity of CXR findings (9).

The net effect of these changes on the reported incidence of PGD3 is unclear. We compared our single-center experience using the 2016 PGD scoring guidelines with results obtained using the 2005 guidelines. Specifically, we compared the incidence, clinical and biological risk factors, and outcomes associated with PGD obtained by using both scoring modalities.

We present the following article in accordance with the STROBE reporting checklist (available at http://dx.doi. org/10.21037/jtd-20-3564).

\section{Methods}

\section{Study population}

We abstracted clinical data from the medical records of 127 consecutive patients who underwent a single or bilateral lung transplant between 1/1/2016-12/31/2018. In addition, we identified 60 consecutive patients ( $\mathrm{n}=30$ women) who underwent transplant between 3/2016-12/2017 and who had preoperative peripheral blood stored in the immunology laboratory at Baylor College of Medicine (BCM); from this cohort, we excluded patients who underwent single-lung or multiorgan transplants and those whose samples were collected $>3$ days before lung transplant. The study was conducted in accordance with the Declaration of Helsinki (as revised in 2013) and was approved by the institutional ethics board at BCM (No. 00000286) with waiver of consent due to the retrospective nature of the study. The data were anonymized for the privacy of the participants.

\section{Blood collection}

The BCM immunology laboratory collects blood samples every 3-6 months from waitlisted patients for HLA profiling. Peripheral blood is collected and allowed to coagulate for 120 minutes at room temperature before centrifugation. The serum is distributed into cryotubes (without evidence of hemolysis or lipemia) and frozen immediately for storage at $-80^{\circ} \mathrm{C}$. We included patients in our study only if blood samples were collected within 3 days before transplant and had undergone $<3$ freeze-thaw cycles.

\section{Measurement of circulating biomarkers}

Frozen serum samples were transferred to the Texas Heart Institute Biorepository Core Laboratory for cytokine quantification via multiplex bead array technique (Bio-Plex, Bio-Rad Laboratories, Hercules, CA, USA) used according to the manufacturer's recommendations. The plates were read with the Luminex MAGPIX with a lower limit of 100 beads per sample per analyte. Data were analyzed using the Bio-plex Results Generator. We measured the concentrations of the following cytokines: basic fibroblast growth factor (FGF), vascular endothelial growth factor, plateletderived growth factor subunit B (PDGF-BB), eotaxin, granulocyte colony-stimulating factor (G-CSF), granulocytemacrophage colony-stimulating factor, interferon gamma (IFN- $\gamma$ ), IFN- $\gamma$-induced protein 10 (IP-10), monocyte chemoattractant protein-1, macrophage inflammatory protein (MIP)- $1 \alpha$ and MIP-1 $\beta$, RANTES, tumor necrosis factor- $\alpha$, and interleukins (IL)-1 $\beta$, IL-1ra, IL2, IL-4, IL-5, IL-6, IL-7, IL-8, IL-9, IL-10, IL-12 (p70), IL13, IL-15, and IL-17A.

Serum concentration of the soluble form of the receptor for advanced glycation end-products (sRAGE) was assessed by enzyme-linked immunoassay (ELISA); the Human RAGE Quantikine ELISA kit (DRG00; R\&D Systems, Minneapolis, MN, USA), which measures the 
total pool of soluble RAGE (10), was used according to the manufacturer's instructions. Briefly, $50 \mu \mathrm{L}$ serum was added to $100 \mu \mathrm{L}$ of buffer, and the mixture was incubated for 2 hours and washed. Secondary antibody was added, and the mixture was incubated again for 2 hours and washed. The substrate solution was added, and the stop solution was added after a 30-minute incubation. Optical density was measured at $450 \mathrm{~nm}$ using the Tecan Infinite 200 PRO plate-reader.

Assays were performed in triplicate for the standard curves and in duplicate for patient samples. A coefficient of variation $<20 \%$ between measurements was used as the acceptance criteria. Personnel performing assays were blinded to the clinical characteristics of the patients.

\section{Determination of PGD grade}

One expert PGD grader (DD) used the ISHLT 2005 and 2016 PGD guidelines and caveats $(1,9)$ to determine PGD at $6,24,48$, and 72 hours after reperfusion. PGD scores for 40 patients (160 scores) were compared to scores determined by another grader to examine inter-observer reliability. Overall agreement between graders was $87.5 \%$ (correlation coefficient, 0.9; $\mathrm{P}<0.0001)$. We herein refer to $\mathrm{PGD}$ as the presence of PGD3 at 48 and/or 72 hours after reperfusion. For extubated patients who had a set $\mathrm{FiO}_{2}$ programmed on a face mask or continuous positive airway pressure or bilevel positive airway pressure device, the standard $\mathrm{PaO}_{2}: \mathrm{FiO}_{2}$ ratio was used in both scoring guidelines if the $\mathrm{FiO}_{2}$ was $>30 \%$. For extubated patients on a nasal cannula, the PGD grade was 0 or 1 depending on the presence of edema on CXR according to the 2005 guidelines. For patients who were extubated and on nasal cannula, the estimated $\mathrm{FiO} 2$ was used according to the 2016 guidelines if the $\mathrm{FiO}_{2}$ was estimated as $>30 \%$. We estimated the $\mathrm{FiO}_{2}$ as follows: $2 \mathrm{~L}=28 \%, 3 \mathrm{~L}$ $=32 \%, 4 \mathrm{~L}=36 \%, 5 \mathrm{~L}=40 \%, 6 \mathrm{~L}=44 \%$, etc. For extubated patients who did not have an arterial blood gas measurement and who had edema on CXR, the PGD grade was calculated according to the $\mathrm{PaO}_{2}: \mathrm{FiO}_{2}$ ratio using a saturation scale as per the 2016 guidelines (9). When such patients were graded according to the 2005 guidelines, a saturation scale was not used, but rather PGD was graded as 0 or 1 , depending on the edema status on CXR.

\section{Measurement of clinical outcomes}

Clinical data on mortality, length of stay, ventilator requirement, and end-organ function were abstracted from patients' records to our lung transplant database. To ensure consistency, we compared the abstracted data with data from UNOS regulatory submissions.

\section{Statistical analysis}

The Fisher exact test was used to compare categorical variables for recipient and donor demographics, donor data, operative variables, and outcomes. Normality was evaluated using the Shapiro-Wilk test. For normally distributed data, statistical assessments were performed using a 2 -tailed Student $t$ test. Nonparametric comparisons were done by Mann Whitney $\mathrm{U}$ and Wilcoxon tests. We used univariable analysis and multivariable logistic regression to estimate the odds ratio for the relationship between clinical risk factors, biomarker levels, PGD development, and mortality. Multivariable analysis was performed with adjustment for clinical factors [age, body mass index (BMI), use of extracorporeal life support (ECLS), presence of pulmonary hypertension, and primary diagnosis]. Stepwise regression and backward regression were used to select the final multivariable regression model. We used the receiver operating characteristics (ROC) of biomarker levels to assess how well the biomarker levels predicted PGD. Differences in progression-free survival curves were estimated using the Kaplan-Meier method, and the absence or presence of mortality was evaluated by log-rank test. $\mathrm{P}$ values were based on a 2 -sided hypothesis. $\mathrm{P}<0.05$ was considered statistically significant. SAS 9.4 was used for statistical analysis, and plots were performed using GraphPad Prism 8.

\section{Results}

\section{Clinical risk factors for PGD}

We included 127 patients who met the inclusion criteria and had complete clinical data for analysis. Using the 2016 scoring guideline, we identified 47 (37\%) patients with PGD3 at 48-72 hours after reperfusion. Patients in the PGD group had a significantly larger BMI than those without PGD (Table 1). The percentage of patients with restrictive lung disease was significantly higher in the PGD group, whereas the percentage of patients with cystic fibrosis was lower. ECLS with either ECMO or cardiopulmonary bypass was used in $89 \%$ of transplant patients and was more common in PGD cases. Based on multivariate analysis, BMI, restrictive lung disease, and ECLS use were associated with PGD, whereas diagnosis of 
Table 1 Demographics and risk factors for patients without and with PGD using 2005 and 2016 guidelines

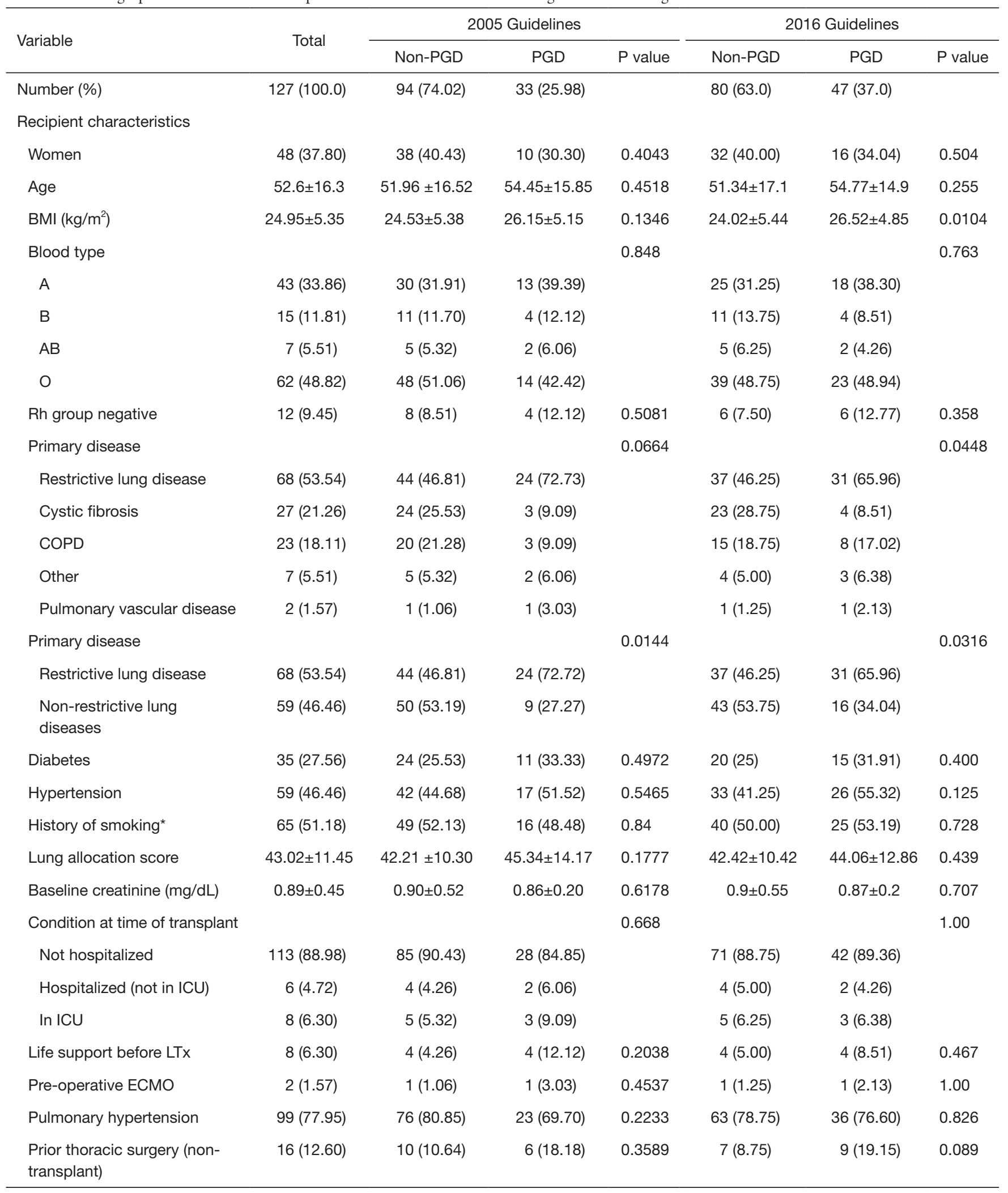

Table 1 (continued) 
Table 1 (continued)

\begin{tabular}{|c|c|c|c|c|c|c|c|}
\hline Variable & Total & \multicolumn{3}{|c|}{2005 Guidelines } & \multicolumn{3}{|c|}{2016 Guidelines } \\
\hline Prior cardiac surgery & $4(3.15)$ & $4(4.26)$ & $0(0.0)$ & 0.572 & $3(3.75)$ & $1(2.13)$ & 1.00 \\
\hline Prior pleurodesis & $7(5.51)$ & $4(4.26)$ & $3(9.09)$ & 0.375 & $4(5.00)$ & $3(6.38)$ & 0.709 \\
\hline Prior lung transplant & $3(2.36)$ & $2(2.13)$ & $1(3.03)$ & 1.000 & $1(1.25)$ & $2(4.26)$ & 0.554 \\
\hline Age (years) & $35.07 \pm 12.2$ & $35.56 \pm 11.99$ & $33.67 \pm 12.88$ & 0.4444 & $35.1 \pm 12.24$ & $35.02 \pm 12.26$ & 0.972 \\
\hline Extended criteria donor ${ }^{\dagger}$ & $45(35.43)$ & $29(30.85)$ & $16(48.48)$ & 0.068 & $25(31.25)$ & $20(42.55)$ & 0.198 \\
\hline Smoker ever $(n=126)^{\ddagger}$ & $67(53.17)$ & $48(51.61)$ & $19(57.58)$ & 0.6852 & $41(51.90)$ & $26(55.3)$ & 0.71 \\
\hline Smoker $>20$ PYH $(n=126)^{\S}$ & $7(5.56)$ & $6(6.45)$ & $1(3.03)$ & 0.6757 & $6(7.59)$ & $1(2.13)$ & 0.2558 \\
\hline Donor after cardiac death & $11(8.66)$ & $5(5.32)$ & $6(18.18)$ & 0.0341 & $4(5.00)$ & $7(14.89)$ & 0.0980 \\
\hline Ex vivo lung perfusion" & $10(7.87)$ & $7(7.45)$ & $3(9.09)$ & 0.7189 & $6(7.50)$ & $4(8.51)$ & 1.00 \\
\hline \multicolumn{8}{|l|}{ Operative characteristics } \\
\hline Type of transplant & & & & 0.825 & & & 0.941 \\
\hline Bilateral & $109(85.83)$ & $80(85.10)$ & $29(87.87)$ & & $68(85.00)$ & $41(87.23)$ & \\
\hline Single & $15(11.81)$ & $12(12.77)$ & $3(9.09)$ & & $10(12.50)$ & $5(10.64)$ & \\
\hline Multi-organ & $3(2.36)$ & $2(2.13)$ & $1(3.03)$ & & $2(2.5)$ & $1(2.13)$ & \\
\hline Type of intraoperative support & & & & 0.0201 & & & 0.017 \\
\hline Total ischemic time (min) & $323 \pm 120.53$ & $315.31 \pm 120.84$ & $346.30 \pm 118.45$ & 0.205 & $317.73 \pm 120.15$ & $332.96 \pm 121.86$ & 0.494 \\
\hline
\end{tabular}

Values are $n(\%)$ or mean \pm SD. ${ }^{*}, n=125+2$ patients' smokeless tobacco (snuff); ${ }^{\dagger}$, one or more of the following: age $>55$ years, anticipated ischemia $>6$ hours, $\mathrm{DCD}, \mathrm{PaO}_{2} / \mathrm{FiO}_{2}<300$, donor is $>20 \mathrm{PYH}$ smoker; ${ }^{\ddagger}, \mathrm{n}=126$ (one unknown donor smoking history); ${ }^{\S}, \mathrm{n}=126$ (one unknown donor smoking history); ", using portable EVLP system. Bilateral, double lung transplant; COPD, chronic obstructive pulmonary disease; ECLS, extracorporeal lung support; ECMO, extracorporeal membrane oxygenation; ICU, intensive care unit; Multiorgan, double lung and additional organs; SD, standard deviation; Single, single lung transplant; 20PYH, 20 pack-year smoking history.

cystic fibrosis was associated with no PGD.

When grading the 127 patients by using the 2005 guidelines, 14 patients were downgraded from PGD3 to PGD1. Thus, only 33 (26\%) patients met the definition for PGD (PGD3 at 48 or 72 hours) (Table 2). The 14 patients who were downgraded were extubated, on nasal-cannula, and received a grade of 1 due to edema on CXR. Patients with 2005 guideline-defined PGD had greater restrictive lung disease and a higher use of donor after cardiac death organs and intraoperative ECLS than those without PGD, although no factors were significant after adjusting for covariables.

The differences in the incidence of PGD3 at individual time points using the 2016 versus 2005 definition were as follows: $45.6 \%$ versus $45.6 \%$ at 6 hours $(\mathrm{P}=1), 33.9 \%$ versus $26.8 \%$ at 24 hours $(\mathrm{P}=0.22), 33.1 \%$ versus $23.6 \%$ 
Table 2 Outcomes in patients without and with PGD using 2005 and 2016 guidelines

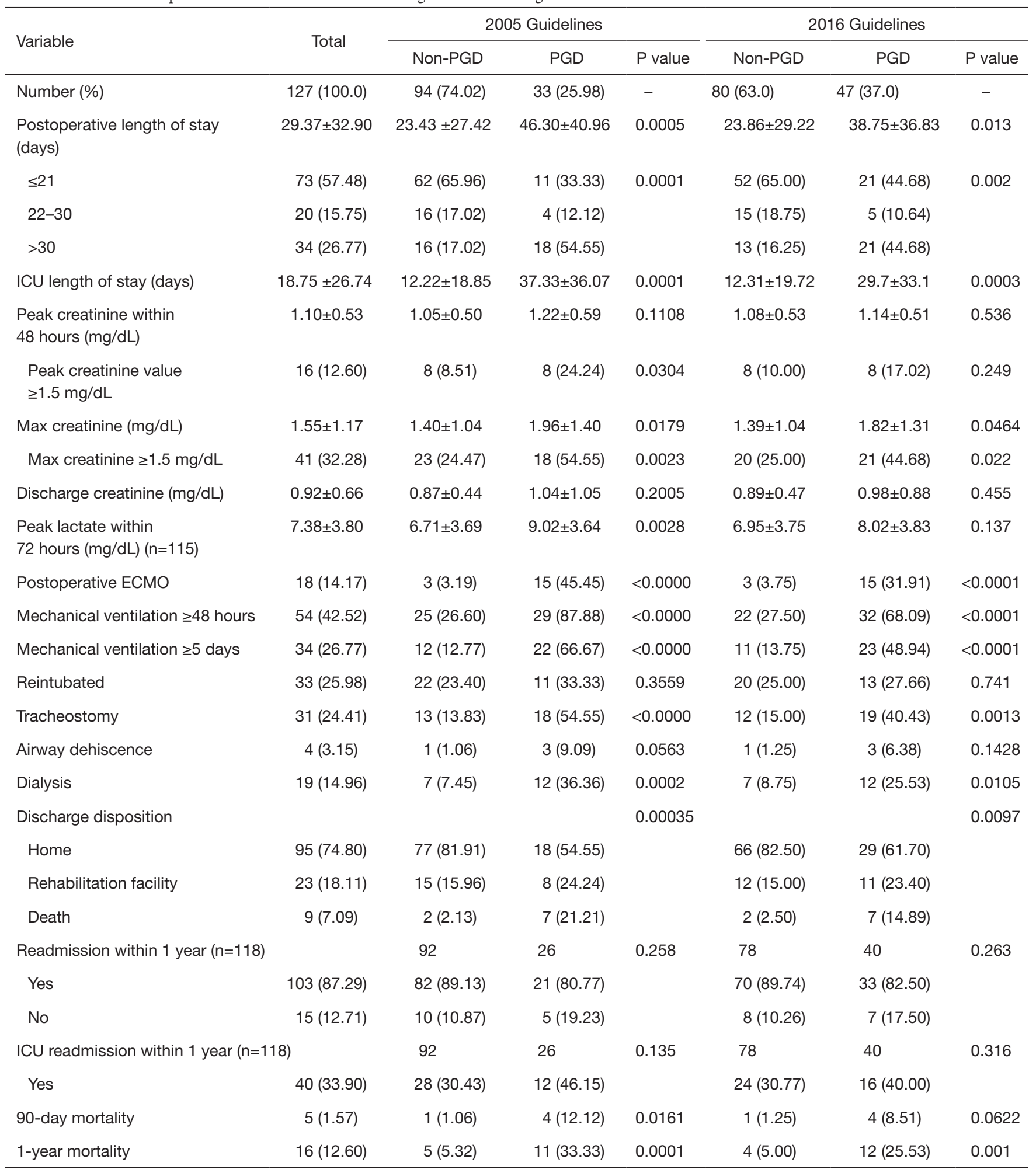

Values are $\mathrm{n}(\%)$ or mean \pm SD. ECMO, extracorporeal membrane oxygenation; ICU, intensive care unit. 


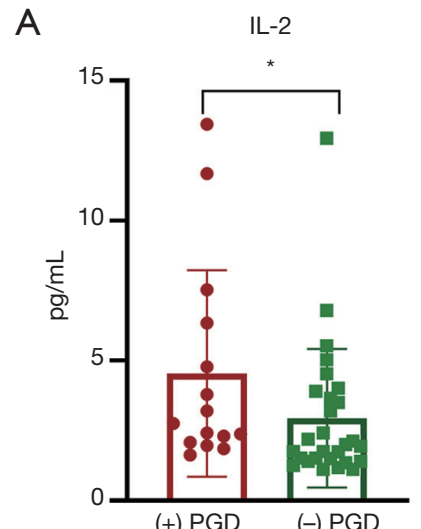

(+) PGD (-) PGD

D

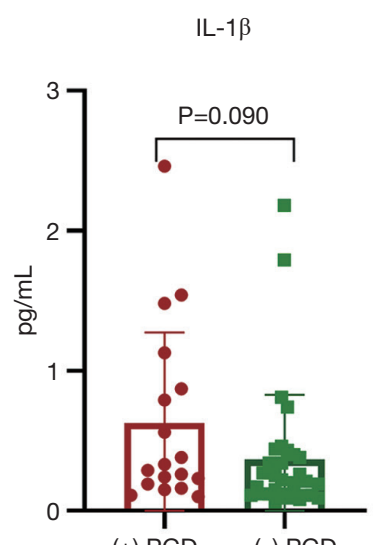

B

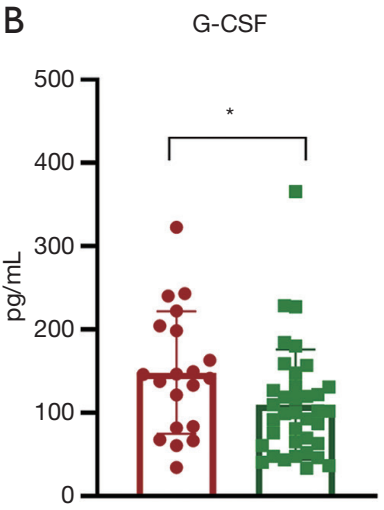

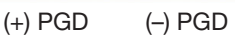

E

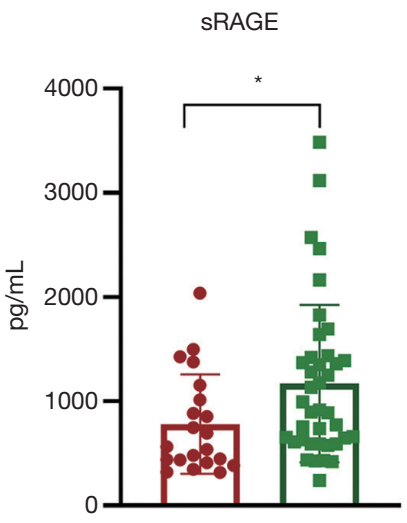

(+) PGD

(-) PGD

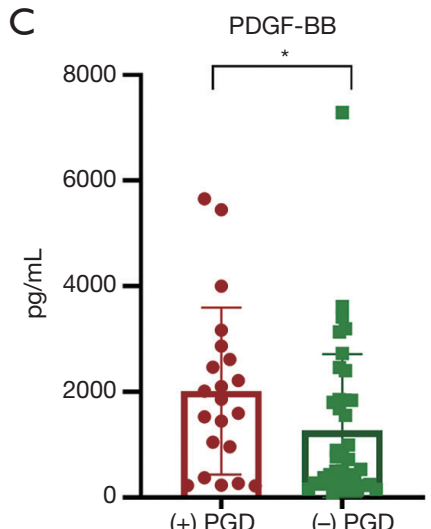

(+) PGD

F

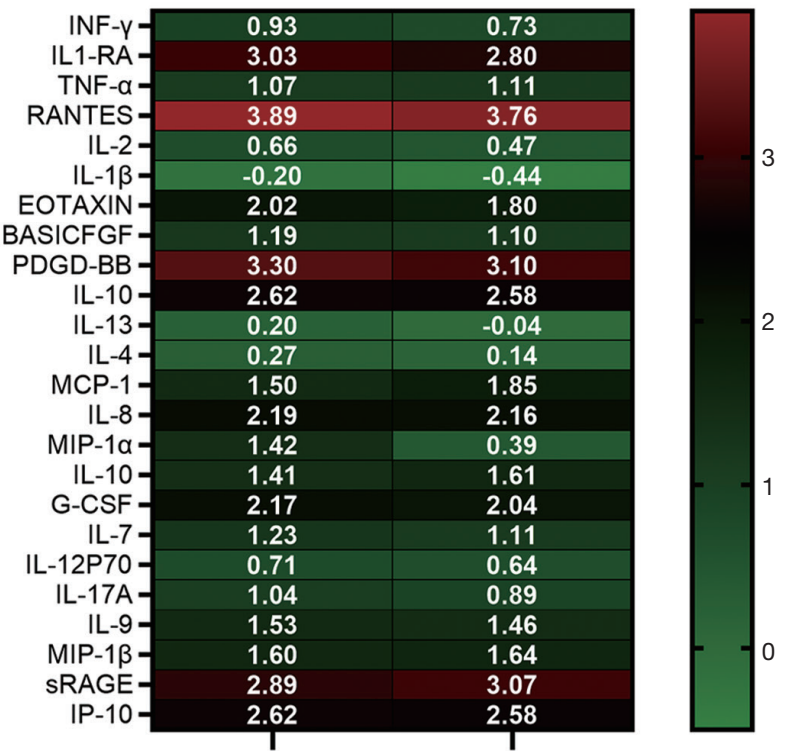

(+) PGD

(-) PGD

Figure 1 Comparison of baseline pro-inflammatory cytokines between patients without (-) and with (+) PGD using the 2016 scoring guidelines. (A) IL-2, (B) G-CSF, (D) PDGF-BB, and (E), IL-1 $\beta$ were elevated in peripheral blood at baseline and (C) sRAGE was decreased in patients who developed PGD. (F) Heat map showing that the $\log 10$ mean concentration of cytokines did not differ in patients with (+) and without (-) PGD. Each symbol represents the mean of an experiment run in duplicate for every patient. *, P<0.05. PGD, primary graft dysfunction; IL, interleukin; G-CSF, granulocyte colony-stimulating factor; PDGF, platelet-derived growth factor.

at 48 hours $(\mathrm{P}=0.09)$, and $30.7 \%$ versus $22.1 \%$ at 72 hours $(\mathrm{P}=0.12)$, respectively. Moreover, the incidence of PGD was $20 \%$ in single lung transplants and $26.8 \%$ in double lung transplants, using the 2005 definition, and $33.3 \%$ versus $37.5 \%$, respectively, using the 2016 definition.

\section{Biomarkers correlated with PGD}

We evaluated baseline serum biomarker concentrations in 60 patients and assessed differences between PGD and non-PGD patients scored with the 2005 and 2016 ISHLT guidelines (Table S1). Clinical characteristics were representative of the larger cohort including more patients with restrictive lung disease and a larger BMI in the PGD group (Table $\mathrm{S} 2$ ).

Using the 2016 ISHLT guidelines, the average levels of IL-2, G-CSF, and PDGF-BB (Figure $1 A, B, C$ ) were significantly higher in the PGD group than in the nonPGD group. A numerical increase without statistical significance was observed in IL-1 $\beta$ (Figure 1D). sRAGE 
levels (Figure $1 E$ ) were higher in the non-PGD group. The $\log 10$ mean concentration of various cytokines (Figure $1 F$ ) showed greater inflammation in PGD samples as depicted by the darker shades (IFN- $\gamma$, IL-1RA, RANTES, EOTAXIN, IP-10, MIP-1A, IL-7), but no significant differences were found between the two groups. Area under the curve analyses for baseline biomarkers associated with PGD using the 2016 guidelines showed that IL-2 had the best predictive capacity for PGD, followed by sRAGE (Figure 2). A multivariate logistic regression analysis using 5 biomarkers (IL-2, G-CSF, PDFG-BB, IL-1 $\beta$, and sRAGE), recipient age, BMI, and diagnosis of restrictive lung disease showed that BMI was the only risk factor significantly associated with PGD. Finally, multivariate logistic regression analysis using only the above-mentioned 5 biomarkers and no clinical factors showed that IL-2 and sRAGE were significantly associated with $\mathrm{PGD}(\mathrm{P}=0.036$ and $\mathrm{P}=0.028$, respectively).

Using the 2005 scoring guidelines, only IL-2 was significantly different at baseline $(6.65$ vs. $3.0 \mathrm{pg} / \mathrm{mL}$, $\mathrm{P}=0.048)$. Higher G-CSF, PDGF-B, and lower sRAGE levels supported the observations in the 2016 cohort, but the differences did not reach significance (Table S1). In the 2005 cohort, multivariate logistic regression analysis using 5 biomarkers (L-2, G-CSF, PDFG-BB, IL-1 $\beta$, and sRAGE), recipient age, $\mathrm{BMI}$, and diagnosis of restrictive lung disease did not show any correlation between baseline biomarkers or clinical risk factors and PGD.

\section{Biomarkers correlated with clinical risk factors}

In addition to exploring differences in baseline biomarkers in patients with PGD, we examined the association between these biomarkers and known PGD risk factors. Using the 2016 scoring guidelines, we analyzed the association between preoperative biomarkers and clinical risk factors for PGD. Figure 3 summarizes the difference between biomarker levels in patients with restrictive lung disease, which showed a higher incidence in patients with PGD. sRAGE levels were significantly lower in patients with restrictive lung disease than in all other patients (766.04 vs. $1,399.71 \mathrm{pg} / \mathrm{mL} ; \mathrm{P}=0.013$ ) but were significantly higher in patients with cystic fibrosis than in patients with other diseases $(1,954.20$ vs. $827.48 \mathrm{pg} / \mathrm{mL} ; \mathrm{P}<0.001)$ (Figure 4). Logistic regression showed that sRAGE was associated with restrictive lung disease. We compared biomarker levels in patients with a $\mathrm{BMI}<$ or $>25 \mathrm{~kg} / \mathrm{m}^{2}$ (Figure 5). sRAGE levels were lower in patients with a higher BMI (740.80 vs.
$1,290.64 \mathrm{pg} / \mathrm{mL} ; \mathrm{P}=0.008)$. Patients with a higher BMI had significantly greater levels of IL-2 (5.70 vs. $2.42 \mathrm{pg} / \mathrm{mL}$; $\mathrm{P}=0.004)$. A linear regression model with stepwise deletion (data not shown) demonstrated that IL-2 was associated with BMI and that sRAGE levels had a significant negative correlation with age (Figure $6 A$ ) and BMI (Figure 6B). No significant correlation was found between other cytokines levels and BMI or age. Patients with lower BMI, who were younger with cystic fibrosis, and who had higher sRAGE levels appeared least likely to develop PGD (Figure S1).

\section{Clinical outcomes correlated with PGD}

No differences were seen in outcomes when comparing assessments using the two guidelines (Table 2). In both guidelines, multiple adverse outcomes were associated with PGD, including extended hospital and ICU stays. The use of dialysis was significantly higher in the PGD group, as were maximum creatinine levels, postoperative ECMO use, prolonged ventilation times ( $>5$ days), and tracheostomy. Furthermore, the PGD group had lower 1-year survival rates using both guidelines (Figure $7 A, B$ ); $25.53 \%$ in the PGD group and $5.0 \%$ of non-PGD patients died at 1 year using the 2016 PGD definition $(\mathrm{P}=0.001)$. Using the 2005 PGD guidelines, $33.33 \%$ in the PGD group and $5.32 \%$ of the non-PGD patients died at 1 year $(\mathrm{P}=0.0001)$ (Table 2).

We assessed the effect of PGD on mortality by using a multiple regression model adjusting for recipient age, sex, BMI, primary disease, lung allocation score, hypertension, donor age, extended criteria donor, donor smoker, type of transplant, use of ECLS, and total ischemic time. PGD was independently associated with 1 -year mortality with the use of both the 2016 [odds ratio $10.7 ; 95 \%$ CI $(2.1,55.2)$ ] and the 2005 guidelines $[15.2 ; 95 \%$ CI $(2.5,93.6)]$. This association was confirmed in a stepwise selection model.

In Table S3, clinical outcomes for patients designated as having PGD according to both 2005 and 2016 guidelines $(\mathrm{n}=33)$ were compared to outcomes in patients who were downgraded to PGD 1 using the 2005 guidelines $(n=14)$. The latter were designated as PGD (PGD3 at 48-72 hours) when using the 2016 guidelines due to strict adherence to saturation ratios and caveats for extubated patients. There were no differences in clinical characteristics between these groups (Table S3). Although the numbers were low, patients who were downgraded in the 2005 guidelines $(n=14)$ had better clinical outcomes and resembled non-PGD patients. They had a significantly reduced length of stay (mean, 20 vs. 46 days, $\mathrm{P}=0.029$, unadjusted), decreased peak lactate 
A

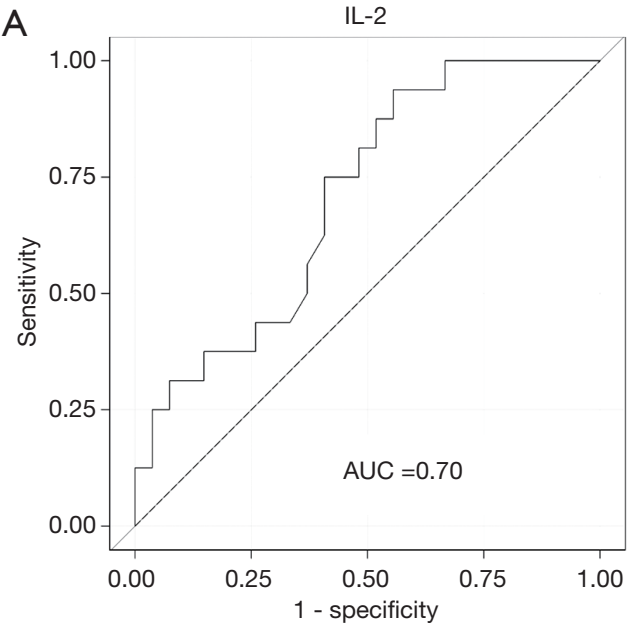

C

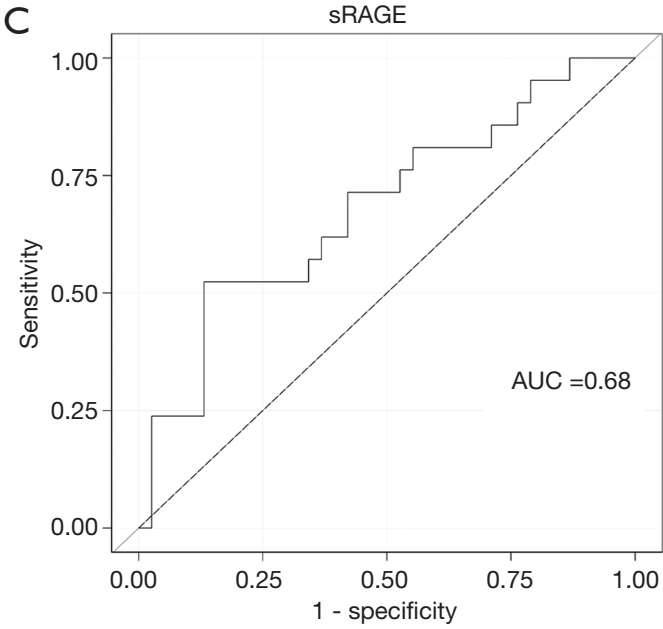

E

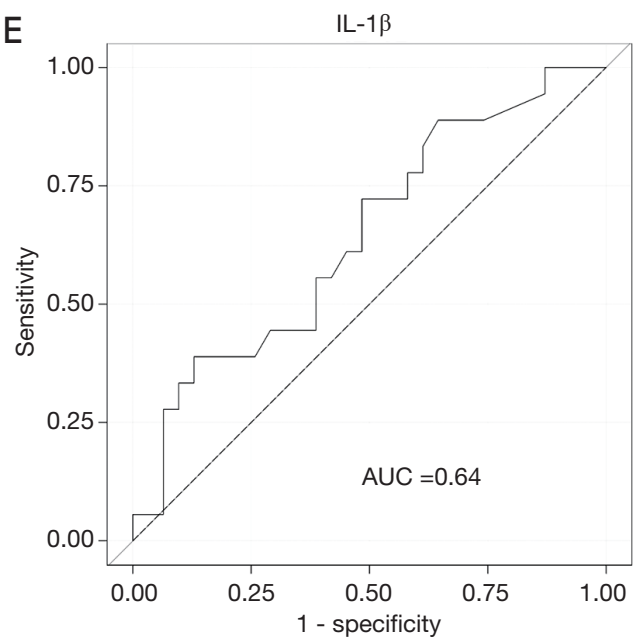

B

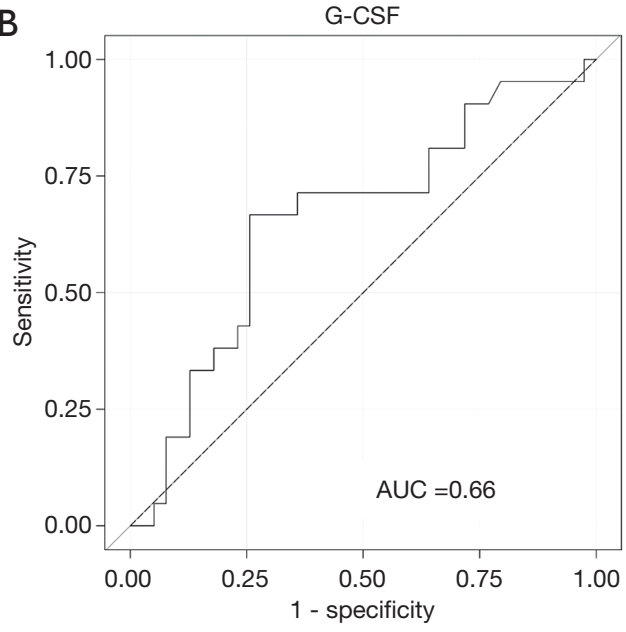

D

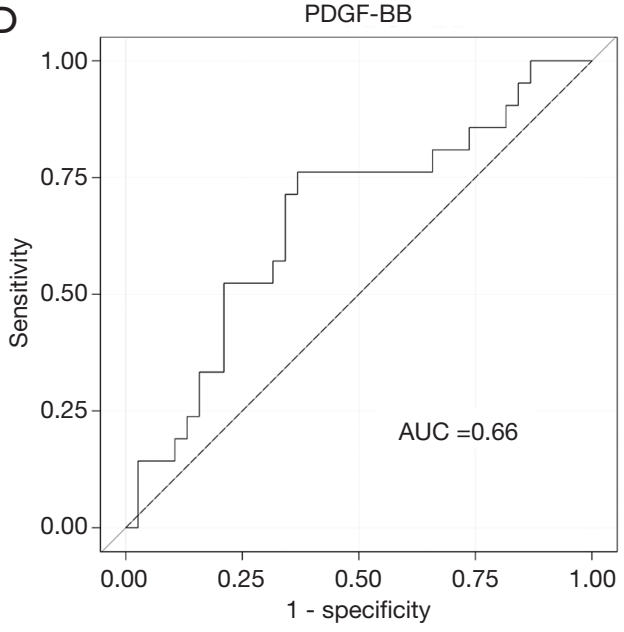

Figure 2 Receiver operating characteristics for baseline cytokines associated with PGD using the 2016 updated guidelines. (A) IL-2, (B) G-CSF, (C) sRAGE, (D) PDGF-BB, and (E) IL-1 $\beta$. AUC, area under the curve; PGD, primary graft dysfunction; IL, interleukin; G-CSF, granulocyte colony-stimulating factor; PDGF, platelet-derived growth factor. 
A

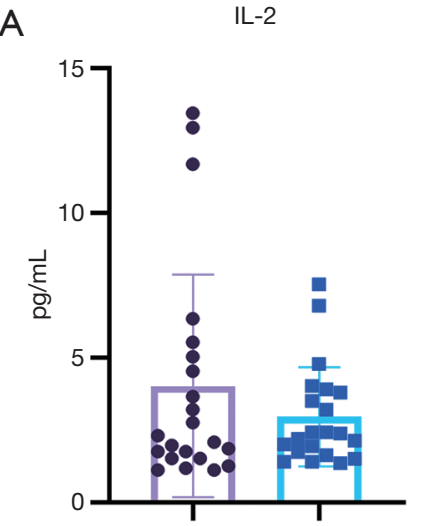

C

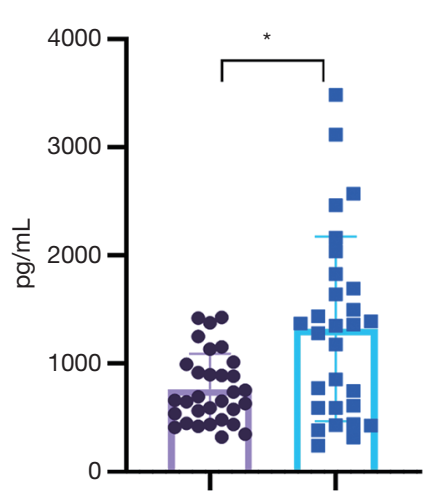

$\mathrm{E}$

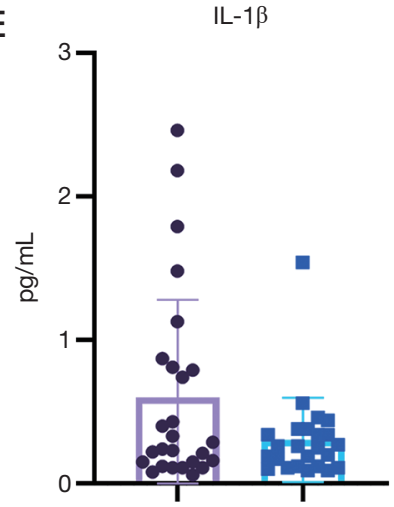

B G-CSF

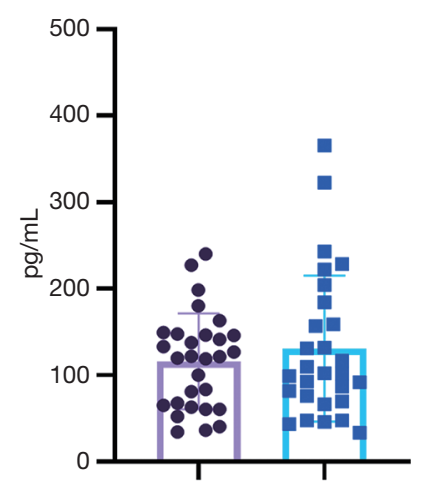

D PDGF-BB

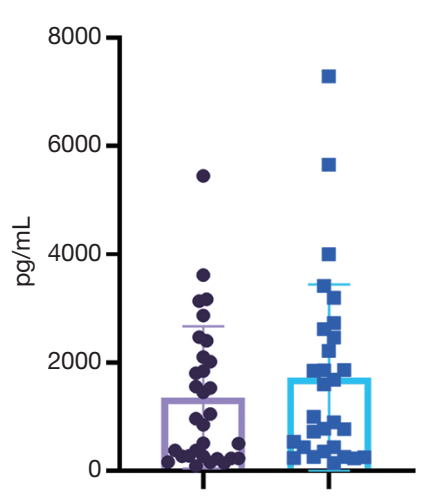

- Restrictive lung disease

- Other diagnoses

Figure 3 Bar plots of serum cytokines in patients diagnosed with restrictive lung disease versus all other baseline diagnoses. (A) IL-2, (B) G-CSF, (C) sRAGE, (D) PDGF-BB, and (E) IL-13. *, P<0.05. IL, interleukin; G-CSF, granulocyte colony-stimulating factor; PDGF, platelet-derived growth factor.

levels (mean, 5.5 vs. $9 \mathrm{mg} / \mathrm{dL}, \mathrm{P}=0.004$, unadjusted), no postoperative ECMO, and lower rates of prolonged ventilation, tracheostomy use ( $7 \%$ vs. $55 \%, \mathrm{P}=0.003)$, and dialysis. Downgraded patients had a numerically lower but non-significant reduction in mortality (1-year mortality, 7\% vs. $33 \%, \mathrm{P}=0.08$, unadjusted).

\section{Discussion}

We examined clinical and biological risk factors and clinical outcomes associated with PGD classified according to the 2005 and the updated 2016 ISHLT guidelines for PGD at a single center. We believe our study will help 
A

IL-2

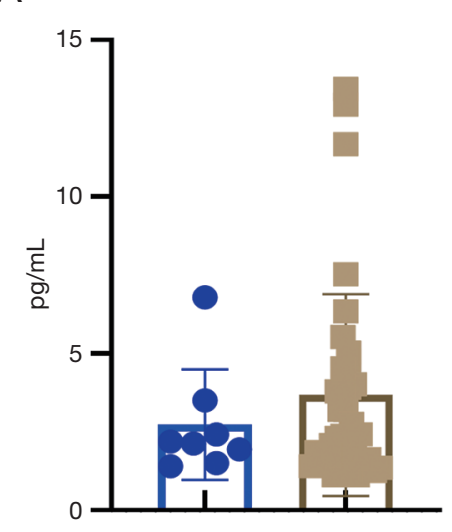

C

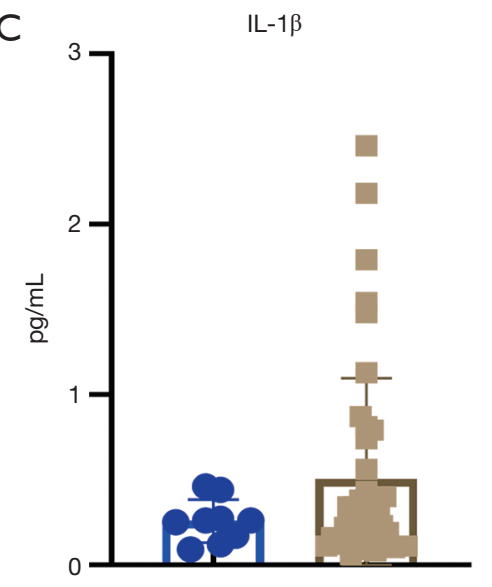

E

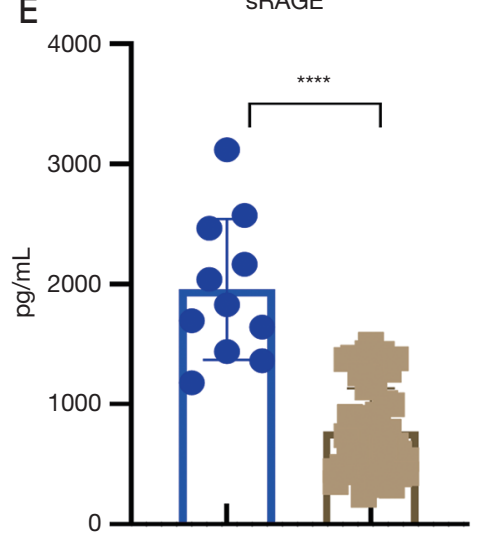

B G-CSF

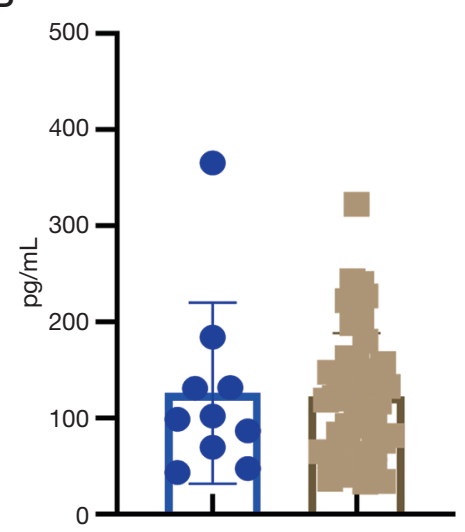

D PDGF-BB

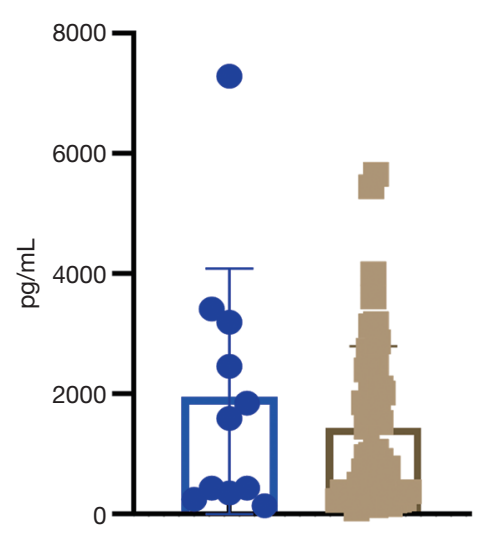

- Cystic fibrosis

Other diagnoses

Figure 4 Bar plots of serum cytokines in patients diagnosed with cystic fibrosis versus all other baseline diagnosis. (A) IL-2, (B) G-CSF, (C) IL-1 $\beta$, (D) PDGF-BB, and (E) sRAGE. ${ }^{* * *}, \mathrm{P}<0.00001$. IL, interleukin; G-CSF, granulocyte colony-stimulating factor; PDGF, plateletderived growth factor.

clinicians gain a better understanding of the effects of the refinements in the updated guidelines in associating PGD with risk factors and clinical outcomes. As expected, we showed that a PGD diagnosis by either guideline resulted in significant morbidity and mortality. This is consistent with previous reports indicating the discriminative ability 

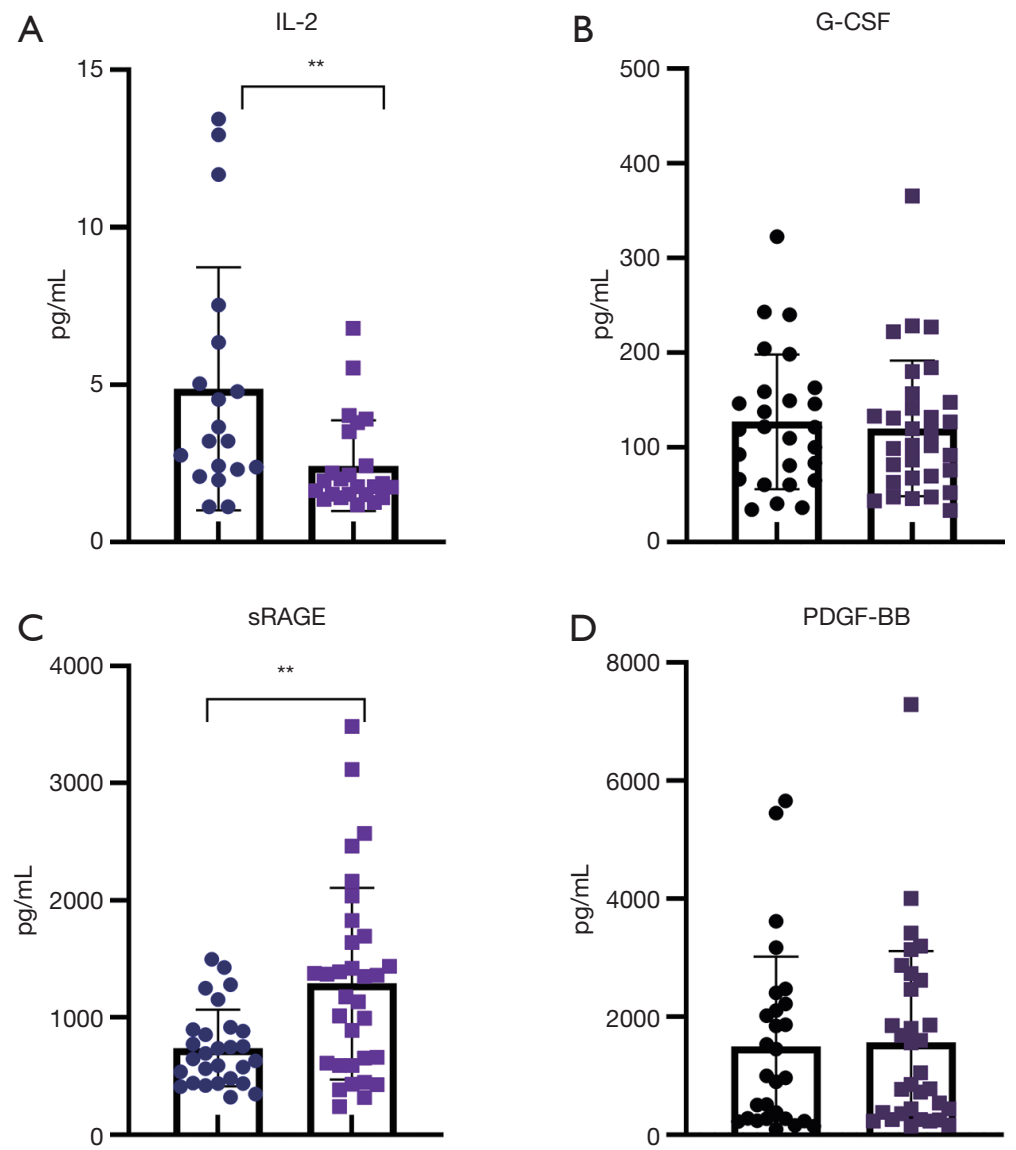

E

$$
\mathrm{IL}-1 \beta
$$

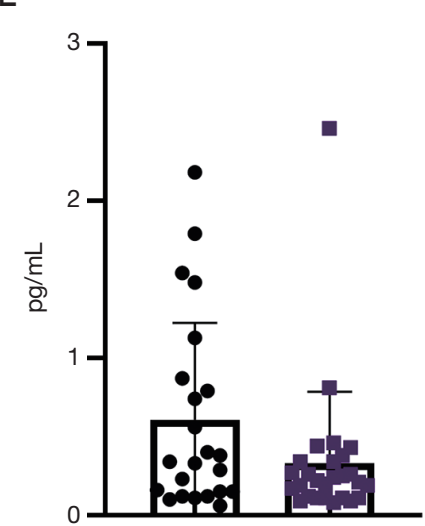

- $\mathrm{BMI}>25$

- $\mathrm{BMl}<25$

Figure 5 Bar plots of serum cytokines in patients with BMI $>25$ versus BMI <25. (A) IL-2, (B) G-CSF, (C) sRAGE, (D) PDGF-BB, and (E) IL$1 \beta .{ }^{* *}, \mathrm{P}<0.001$. BMI, body mass index; IL, interleukin; G-CSF, granulocyte colony-stimulating factor; PDGF, platelet-derived growth factor.

of arterial oxygen partial pressure to fractional inspired oxygen ratios $\left(\mathrm{PaO}_{2} / \mathrm{FiO}_{2}\right)$ at $48-72$ hours post-transplant to show differences in mortality regardless of ventilation strategy $(6,11)$. More cases of PGD were diagnosed by using the 2016 guidelines, mostly due to the clarified grading criteria for extubated patients that were associated with greater sensitivity in identifying risk factors for PGD such as ECLS use, recipient BMI, and diagnosis of restrictive lung disease. The limited numbers and decreased sensitivity of these factors in the 2005 guidelines resulted in 

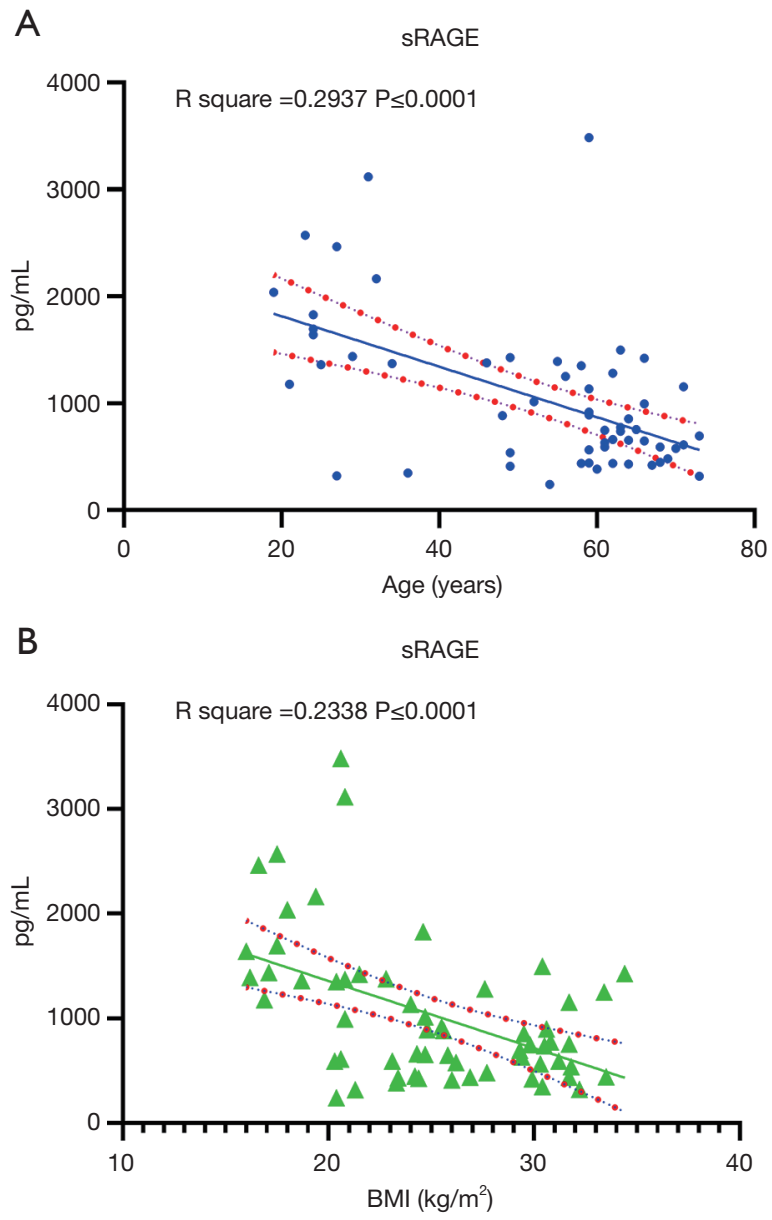

Figure 6 Linear regression graphs showing correlation between sRAGE and patient clinical characteristics. (A) Correlation between sRAGE levels and age, (B) correlation between sRAGE and BMI. BMI, body mass index.

a weaker association in our single-center cohort. Biological risk factors were also more prevalent in the 2016 cohort than in the 2005 cohort, although no biomarkers were independently associated with PGD after adjusting for clinical risk factors.

In 2013, the Lung Transplant Outcomes Group (LTOG), using the 2005 PGD scoring system (5), reported a $16.8 \%$ incidence of PGD3 at 48 or 72 hours, which was strongly associated with an increased 90-day and 1-year mortality (5). Using the 2005 PGD scoring system, we reported a $26 \%$ incidence of PGD3 in a smaller single center cohort. However, using the updated 2016 PGD guidelines with specific grading protocols for extubated patients, we found a 37\% incidence of PGD3 at 48-72 hours. These rates were higher than those reported by the LTOG in 2013, but our 1-year survival rates were similar. Extended criteria donor was used in $35 \%$ of cases and ECLS in $89 \%$ of cases. Both factors, along with the updated guidelines, could have led to a higher PGD rate. Of note, our ECLS practice has evolved from use of cardiopulmonary bypass, a known risk factor for PGD, to the use of ECMO, considered by some to reduce PGD $(5,12)$. In general, we use ECLS to improve intraoperative hemodynamics and exposure when needed.

Consistent with previous reports, we found that a designation of PGD according to the updated 2016 consensus was significantly associated with several adverse events including prolonged hospital and ICU length of stays. In addition, patients with PGD had a prolonged intubation period and required a tracheostomy more often than did those without PGD. Moreover, PGD patients were more likely to require dialysis and postoperative ECMO and to have a peak creatinine $\geq 1.5 \mathrm{mg} / \mathrm{dL}$ within 48 hours of reperfusion. Our findings support those of others suggesting considerable cross talk between the lungs and kidney (13). Impaired gas exchange and increased cytokines from lung injury can lead to renal microvascular dysregulation, apoptosis, and endothelial dysfunction. The lack of renal fluid and solute clearance can exacerbate pulmonary dysfunction and edema.

In our study, 90-day mortality for patients with PGD was only $8.5 \%$, which is substantially better than previous reports $(5,14)$. However, the likelihood of dying in the hospital was 6-fold higher in those with PGD than in those without it. In addition, the 1-year mortality was $25.5 \%$ for PGD patients and $5 \%$ for those without it. These findings were also seen with the 2005 guidelines, suggesting a similar impact of PGD by either scoring system (5). Importantly, our analysis did demonstrate that the subgroup of patients who were designated as PGD3 at 48-72 hours while extubated fared better than those who were intubated. However, this small subgroup analysis requires further study as it suggests that patients who are designated as PGD3 according to the 2016 guidelines may have a better prognosis if they are extubated at 48-72 hours. Whether this reflects a mechanistic link between lower barotrauma and better lung healing from the avoidance of mechanical ventilation or is simply a marker of less severe lung injury requires further investigation.

Previous reports have linked baseline biomarker levels in recipients with the risk of PGD using early PGD scoring definitions (15). In evaluating pre-transplant levels of circulating cytokines and chemokines, we noted a trend toward greater inflammation in patients who eventually 


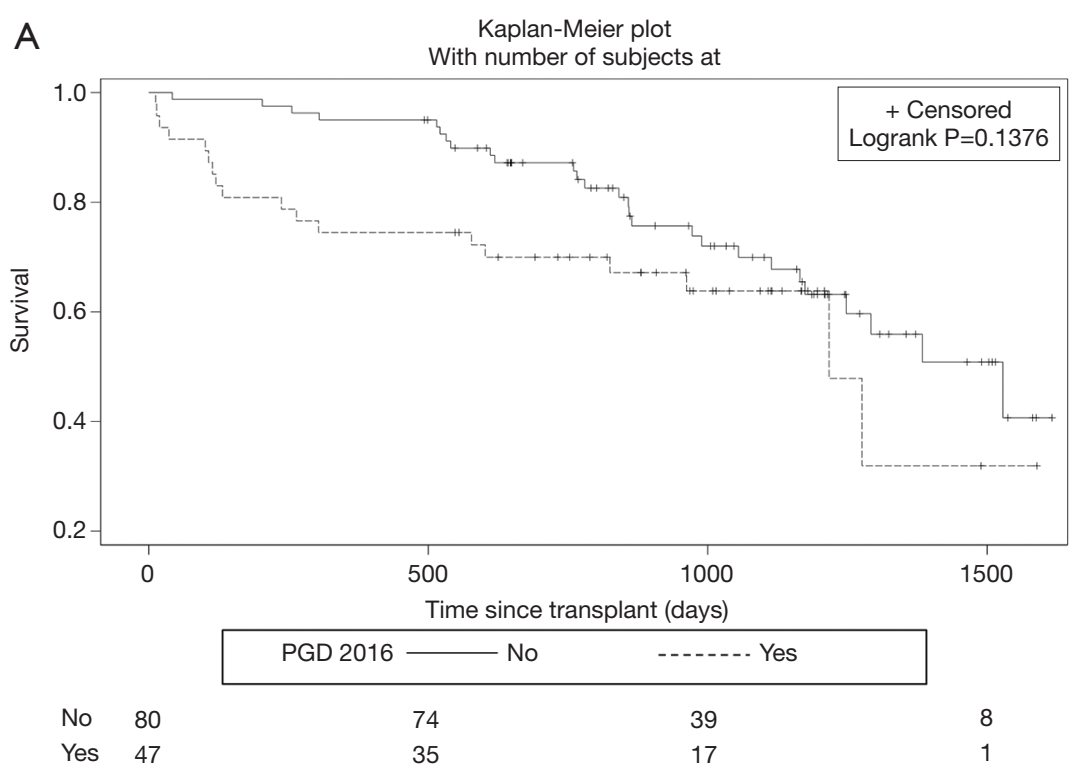

B

Kaplan-Meier plot

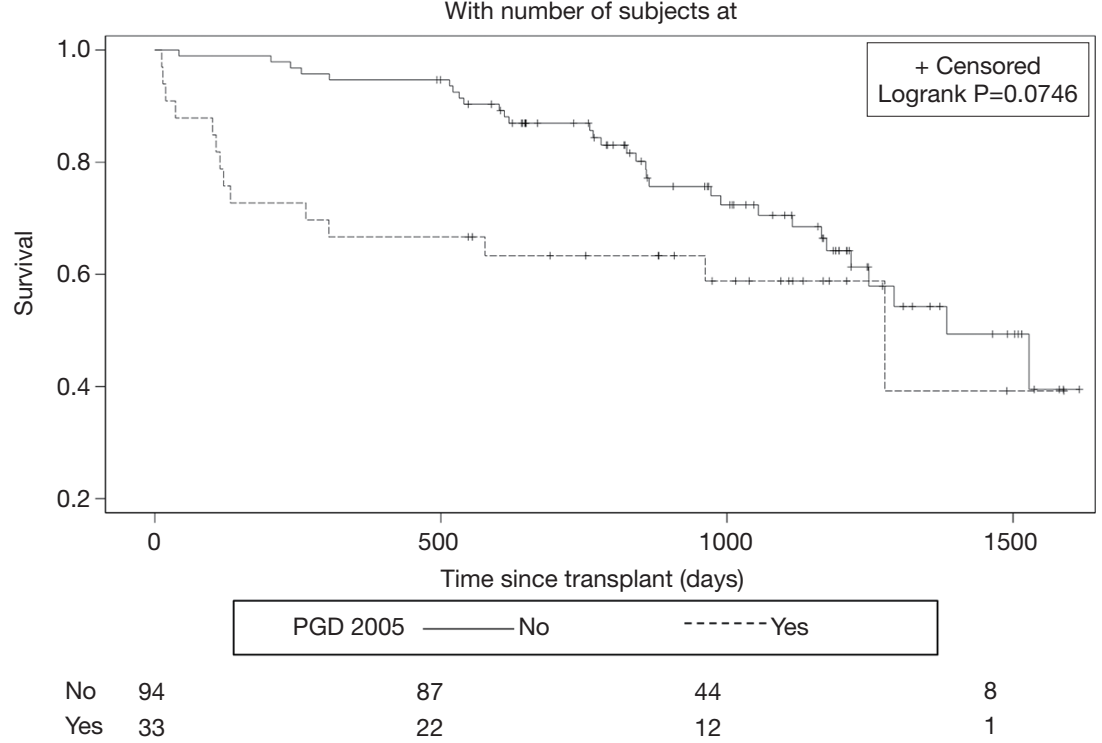

Figure 7 Kaplan-Meier plots of survival estimate for patients without and with PGD. (A) Survival estimate using the 2016 scoring guidelines, (B) survival estimate using the 2005 scoring guidelines. PGD, primary graft dysfunction.

developed PGD (Figure 1F). Three biomarkers were significantly associated with PGD on univariate analysis: pre-transplant IL-2 and PDGF-BB levels were higher and sRAGE levels were lower. ROC and multivariate analysis of cytokines suggested that IL-2 and sRAGE were the most predictive of PGD. Only IL-2 was predictive of PGD with the 2005 guidelines, suggesting the updated guidelines may be more sensitive at detecting preoperative molecular risk factors. However, no biomarker was independently associated with PGD development after adjusting for clinical risk factors.

PGD pathophysiology involves an ischemia-reperfusionmediated injury to alveolar, endothelial, and interstitial cells, followed by the migration of polymorphonuclear neutrophils from the pulmonary circulation into the interstitium and airways $(16,17)$. Immune responses to pulmonary injury ultimately exacerbate lung damage and lead to the development of clinical PGD. It is reasonable 
to hypothesize that a recipient's baseline inflammatory state may predict PGD development. Although the underlying mechanism is unclear, both innate and adaptive immunity rely on cytokine signaling to enhance activity. A greater baseline milieu of cytokine signals could fuel immunerelated events leading to PGD. For example, IL-2 enhances immune activity via multiple pathways (18), so patients with elevated IL-2 levels may be primed for a heightened inflammatory response that could exacerbate PGD injury.

Levels of sRAGE were lower in patients who developed PGD. RAGE is a transmembrane receptor of the immunoglobulin superfamily that is highly expressed in the lungs (19). RAGE activation modulates cell signaling that enhances inflammatory responses. Furthermore, Christie et al. showed that sRAGE levels were higher 6 and 24 hours after reperfusion in patients who developed PGD, although baseline measurements were not reported (20). Pelaez et al. reported that higher sRAGE concentrations in donor levels before transplant and recipient levels after transplantation were associated with PGD development (21), but again pretransplant levels were not measured in recipients. Others have shown that elevated sRAGE levels early during reperfusion were predictive of increased PGD and adverse events using the 2005 guidelines $(20,22)$.

It is challenging to explain why patients at risk of PGD would have lower levels of sRAGE. One possible explanation is, as we and others have shown, that sRAGE was decreased in patients with idiopathic pulmonary fibrosis (which was associated with PGD in our study) as compared with controls (23-25). Interestingly, sRAGE levels may be higher in patients with COPD (26) and in cystic fibrosis (27); in our study, only $9 \%$ of patients with cystic fibrosis and less than $20 \%$ of COPD patients developed PGD. This could explain the higher levels of sRAGE in non-PGD patients.

Using the 2016 consensus guidelines, we found an association between several baseline biomarkers and known PGD risk factors. Patients with restrictive lung disease had significantly lower levels of sRAGE than did patients with other diseases. Patients with a BMI $>25 \mathrm{~kg} / \mathrm{m}^{2}$ had significantly lower sRAGE levels and higher IL-2. These findings suggest that clinical risk factors for PGD such as increased BMI and underlying disease may contribute to the inflammation that primes patients for developing PGD under the right circumstances.

This retrospective single-center study has several limitations. To mitigate the biases of retrospective data collection, the data were cross-referenced to data collected for regulatory reasons and were deemed consistent and accurate. Also, PGD was scored by an expert single grader with access to all relevant respiratory, blood gas, and CXR data. However, the timing of the collection of arterial blood gas samples, CXRs, and ventilator parameters was not standardized. Our comparison of the two guidelines resulted in differences related to the grading of extubated patients. We did not have ECMO cases for non-hypoxic indications or ECMO with clear CXR, which would be considered un-gradable in the 2016 guidelines. Thus, we cannot make conclusions about the effects of the updated ECMO grading on the reported incidence and risk factors of PGD. Moreover, serum samples were stored at $-80{ }^{\circ} \mathrm{C}$ and may have deteriorated over time. The number of samples was limited due to budget constraints and availability of those obtained within 3 days of transplant. We excluded the few patients who underwent multi-organ and single-lung transplants from the study cohort of 60 patients to yield a more homogeneous group. However, clinical characteristics of this group of 60 patients were representative of the larger cohort including more patients with restrictive lung disease and larger BMIs in the PGD group (Table S2). Also, clinical outcomes were representative of the larger cohort including greater adverse events in the PGD group (Table S4). Multiplex experiments were used because of their potential to yield a broader molecular phenotype using a low sample volume; however, they are subject to false positives due to the multiple comparisons used in the statistical analysis. Also, four biomarkers were below the detection range (GM-CSF, IL-5, vascular endothelial growth factor, IL-15), making it difficult to interpret their relationship with PGD.

The grading of PGD in single lung transplants is controversial. The native contralateral lung could protect the allograft from causing systemic hypoxia; however, it could also exacerbate hypoxia. Previous analysis identified single lung transplants as an important risk factor for PGD (5). The 2016 ISHLT consensus guidelines suggest reporting the rates individually, as done in Table 1, but they do not suggest changing the scoring of PGD in single lungs. For the pooled analysis of outcomes and risk factors for PGD, we did not exclude single lung transplants as they represent $12 \%$ of the overall cohort and could substantially bias our results. We did carefully review each single lung CXR and grade the PGD according to the systemic $\mathrm{PaO}_{2}$ or saturation scale in accordance with the guidelines. Importantly, we did exclude single lung transplants from our analysis of the effect of baseline cytokine levels on the development of PGD. This was done to reduce heterogeneity in the smaller subgroup analysis 
and to reduce the potential bias of residual inflammatory contribution from the remaining contralateral lung.

In conclusion the current analysis highlights improvements in the updated PGD scoring guidelines, which still predict substantial adverse effects of PGD on patient outcomes but also increase the detection of PGD and clinical and molecular risk factors. Thus, the new scoring system is more sensitive, which has important research implications and may improve the ability to detect risk factors, but the clinical implications of having a sensitive scoring system may not be as severe if the patient remains extubated at 48-72 hours post-transplant.

We recommend using the updated 2016 PGD scoring guidelines in all lung transplant recipients, independent of intubation status, as a vital performance and research metric. Reporting and analysis of these scores could lead to improved detection, prevention, and management of PGD. The inverse correlations between sRAGE levels and PGD and between sRAGE and clinical risk factors for PGD are novel findings that warrant further study. Despite the potential mechanistic insight gained from preoperative biomarkers and the potential for identifying molecular phenotypes of PGD, clinical risk factors alone appear to be sufficient for predicting patients at greater risk.

\section{Acknowledgments}

The authors thank Rebecca Bartow, PhD, of the Department of Scientific Publications at the Texas Heart Institute, for editorial assistance.

Funding: This research was supported by the Texas Heart Institute and Baylor College of Medicine in data collection, the Roderick Duncan MacDonald General Research Fund, and the JLH Foundation for Lung Transplant Research.

\section{Footnote}

Reporting Checklist: The authors have completed the STROBR reporting checklist. Available at http://dx.doi. org/10.21037/jtd-20-3564

Data Sharing Statement: Available at http://dx.doi. org/10.21037/jtd-20-3564

Conflicts of Interest: All authors have completed the ICMJE uniform disclosure form (available at http://dx.doi. org/10.21037/jtd-20-3564). GL reports grants from Transmedics, Inc., during the conduct of the study; grants from Abiomed, related travel/housing reimbursement from Transmedics, Inc., grants from Medtronic, grants from Abbott, outside the submitted work. The other authors have no conflicts of interest to declare.

Ethical Statement: The authors are accountable for all aspects of the work in ensuring that questions related to the accuracy or integrity of any part of the work are appropriately investigated and resolved. The study was conducted in accordance with the Declaration of Helsinki (as revised in 2013). The study was approved by the institutional ethics board at Baylor College of Medicine (No. 00000286) with waiver of consent due to the retrospective nature of the study. The data was anonymized for the privacy of the participants.

Open Access Statement: This is an Open Access article distributed in accordance with the Creative Commons Attribution-NonCommercial-NoDerivs 4.0 International License (CC BY-NC-ND 4.0), which permits the noncommercial replication and distribution of the article with the strict proviso that no changes or edits are made and the original work is properly cited (including links to both the formal publication through the relevant DOI and the license). See: https://creativecommons.org/licenses/by-nc-nd/4.0/.

\section{References}

1. Christie JD, Carby M, Bag R, et al. Report of the ISHLT Working Group on Primary Lung Graft Dysfunction part II: definition. A consensus statement of the International Society for Heart and Lung Transplantation. J Heart Lung Transplant 2005;24:1454-9.

2. Prekker ME, Nath DS, Walker AR, et al. Validation of the proposed International Society for Heart and Lung Transplantation grading system for primary graft dysfunction after lung transplantation. J Heart Lung Transplant 2006;25:371-8.

3. Whitson BA, Nath DS, Johnson AC, et al. Risk factors for primary graft dysfunction after lung transplantation. J Thorac Cardiovasc Surg 2006;131:73-80.

4. Samano MN, Fernandes LM, Baranauskas JC, et al. Risk factors and survival impact of primary graft dysfunction after lung transplantation in a single institution. Transplant Proc 2012;44:2462-8.

5. Diamond JM, Lee JC, Kawut SM, et al. Clinical risk factors for primary graft dysfunction after lung transplantation. Am J Respir Crit Care Med 2013;187:527-34. 
6. Cantu E, Shah RJ, Lin W, et al. Oxidant stress regulatory genetic variation in recipients and donors contributes to risk of primary graft dysfunction after lung transplantation. J Thorac Cardiovasc Surg 2015;149:596-602.

7. Hamilton BC, Kukreja J, Ware LB, et al. Protein biomarkers associated with primary graft dysfunction following lung transplantation. Am J Physiol Lung Cell Mol Physiol 2017;312:L531-L541.

8. Porteous MK, Lee JC. Primary graft dysfunction after lung transplantation. Clin Chest Med 2017;38:641-54.

9. Snell GI, Yusen RD, Weill D, et al. Report of the ISHLT Working Group on Primary Lung Graft Dysfunction, part I: Definition and grading-A 2016 Consensus Group statement of the International Society for Heart and Lung Transplantation. J Heart Lung Transplant 2017;36:1097-103.

10. Mahajan N, Dhawan V, Malik S, et al. Serum levels of soluble receptor for advanced glycation end products (sRAGE) in Takayasu's arteritis. Int J Cardiol 2010;145:589-91.

11. Cantu E, Diamond JM, Suzuki Y, et al. Quantitative Evidence for Revising the Definition of Primary Graft Dysfunction after Lung Transplant. Am J Respir Crit Care Med 2018;197:235-43.

12. Hoetzenecker K, Benazzo A, Stork T, et al. Bilateral lung transplantation on intraoperative extracorporeal membrane oxygenator: An observational study. J Thorac Cardiovasc Surg 2020;160:320-327.e1.

13. Husain-Syed F, Slutsky AS, Ronco C. Lung-kidney crosstalk in the critically ill patient. Am J Respir Crit Care Med 2016;194:402-14.

14. Whitson BA, Prekker ME, Herrington CS, et al. Primary graft dysfunction and long-term pulmonary function after lung transplantation. J Heart Lung Transplant 2007;26:1004-11.

15. Allen JG, Lee MT, Weiss ES, et al. Preoperative recipient cytokine levels are associated with early lung allograft dysfunction. Ann Thorac Surg 2012;93:1843-9.

16. Kreisel D, Goldstein DR. Innate immunity and organ

Cite this article as: Daoud D, Chacon Alberty L, Wei Q, Hochman Mendez C, Virk MHM, Mase J, Jindra P, Cusick M, Choi H, Debolske N, Sampaio LC, Taylor DA, Loor G. Incidence of primary graft dysfunction is higher according to the new ISHLT 2016 guidelines and correlates with clinical and molecular risk factors. J Thorac Dis 2021;13(6):3426-3442. doi: $10.21037 /$ jtd-20-3564 transplantation: focus on lung transplantation. Transpl Int 2013;26:2-10.

17. Laubach VE, Kron IL. Pulmonary inflammation after lung transplantation. Surgery 2009;146:1-4.

18. Spolski R, Li P, Leonard WJ. Biology and regulation of IL-2: from molecular mechanisms to human therapy. Nat Rev Immunol 2018;18:648-59.

19. Creagh-Brown BC, Burke-Gaffney A, Evans TW. sRAGE: a useful biomarker in acute lung injury? Crit Care Med 2011;39:589-90.

20. Christie JD, Shah CV, Kawut SM, et al. Plasma levels of receptor for advanced glycation end products, blood transfusion, and risk of primary graft dysfunction. Am J Respir Crit Care Med 2009;180:1010-5.

21. Pelaez A, Force SD, Gal AA, et al. Receptor for advanced glycation end products in donor lungs is associated with primary graft dysfunction after lung transplantation. Am J Transplant 2010;10:900-7.

22. Calfee CS, Budev MM, Matthay MA, et al. Plasma receptor for advanced glycation end-products predicts duration of ICU stay and mechanical ventilation in patients after lung transplantation. J Heart Lung Transplant 2007;26:675-80.

23. Machahua C, Montes-Worboys A, Planas-Cerezales L, et al. Serum AGE/RAGEs as potential biomarker in idiopathic pulmonary fibrosis. Respir Res 2018;19:215.

24. Konishi K, Gibson KF, Lindell KO, et al. Gene expression profiles of acute exacerbations of idiopathic pulmonary fibrosis. Am J Respir Crit Care Med 2009;180:167-75.

25. Habermann AC, Gutierrez AJ, Bui LT, et al. Singlecell RNA sequencing reveals profibrotic roles of distinct epithelial and mesenchymal lineages in pulmonary fibrosis. Sci Adv 2020;6:eaba1972.

26. Wu L, Ma L, Nicholson LF, et al. Advanced glycation end products and its receptor (RAGE) are increased in patients with COPD. Respir Med 2011;105:329-36.

27. Beucher J, Boelle PY, Busson PF, et al. AGER -429T/C is associated with an increased lung disease severity in cystic fibrosis. PloS One 2012; 7:e41913. 


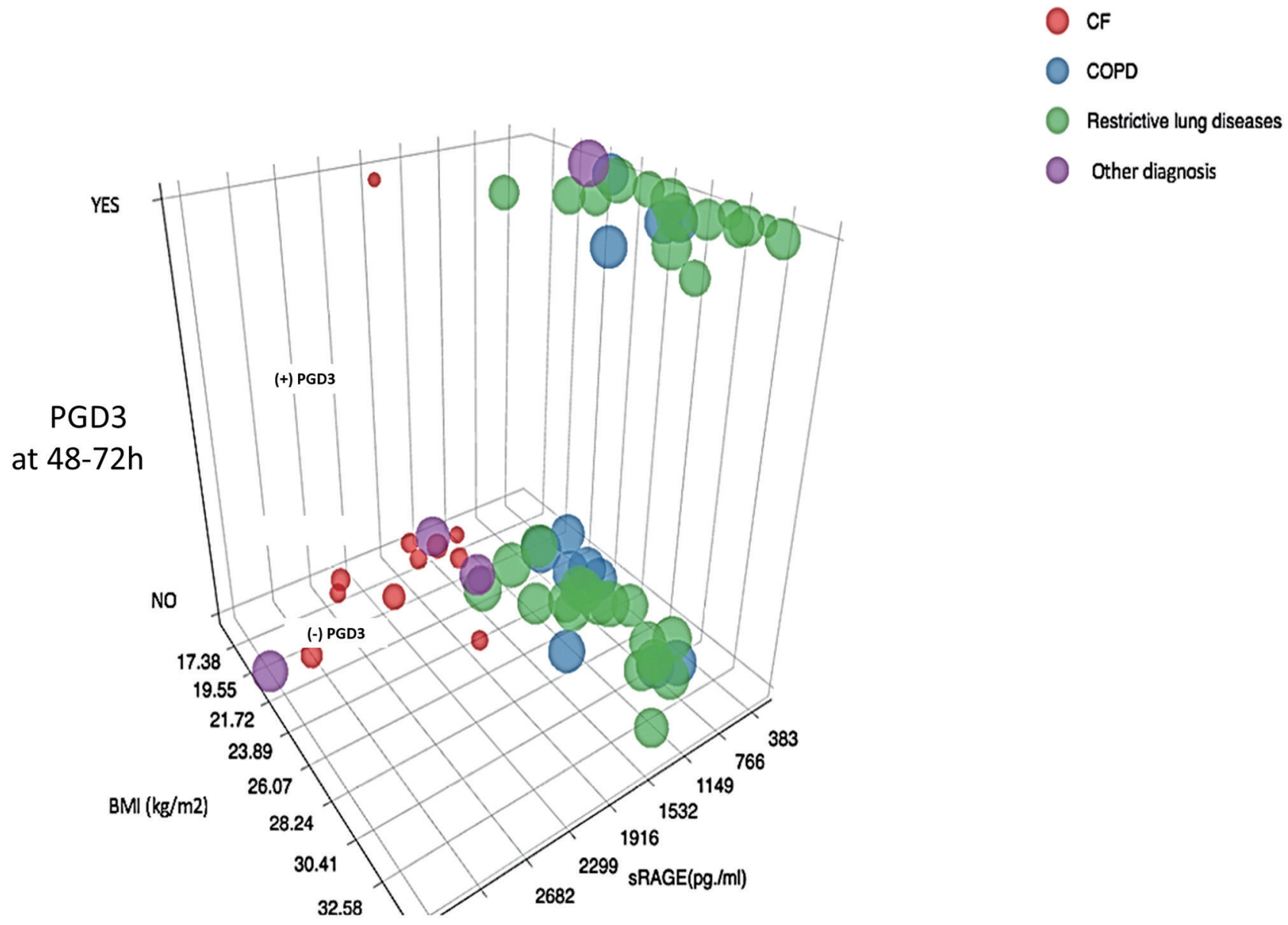

Figure S1 Relationship between underlying disease, body mass index (BMI), age (circle size), and presence or absence of PGD3 and sRAGE concentration. CF, cystic fibrosis; COPD, chronic obstructive pulmonary disease. 
Table S1 Baseline cytokines using 2005 and 2016 guidelines (n=60; HLA samples)

\begin{tabular}{|c|c|c|c|c|c|c|}
\hline Cytokines & \multicolumn{3}{|c|}{2005 Guidelines } & \multicolumn{3}{|c|}{2016 Guidelines } \\
\hline MIP-1B & $41.34 \pm 53.42$ & $44.15 \pm 29.18$ & 0.208 & $43.41 \pm 57.58$ & $39.36 \pm 25.85$ & 0.540 \\
\hline IL-6 & $7.91 \pm 18.17$ & $2.31 \pm 3.87$ & 0.703 & $7.59 \pm 17.77$ & $4.62 \pm 9.25$ & 0.591 \\
\hline IL-1RA & $761.51 \pm 1,847.38$ & $842.36 \pm 1,961.00$ & 0.442 & $626.86 \pm 1,454.63$ & $1,065.47 \pm 2,455.67$ & 0.296 \\
\hline Rantes & $6,035.28 \pm 8,018.89$ & $7,970.43 \pm 9,654.13$ & 0.207 & $5,736.44 \pm 8,027.56$ & $7,794.98 \pm 9,060.21$ & 0.158 \\
\hline IL-2 & $3.00 \pm 2.30$ & $6.65 \pm 6.45$ & 0.048 & $2.94 \pm 2.47$ & $4.55 \pm 3.69$ & 0.018 \\
\hline IL-1 $\beta$ & $0.42 \pm 0.55$ & $0.59 \pm 0.55$ & 0.351 & $0.37 \pm 0.46$ & $0.63 \pm 0.65$ & 0.090 \\
\hline Eotaxin & $77.19 \pm 93.28$ & $77.66 \pm 83.15$ & 0.766 & $63.16 \pm 77.53$ & $103.57 \pm 107.42$ & 0.196 \\
\hline IP-10 & $366.63 \pm 440.70$ & $473.87 \pm 465.75$ & 0.421 & $378.85 \pm 471.79$ & $415.43 \pm 400.43$ & 0.403 \\
\hline IL-13 & $1.08 \pm 1.04$ & $1.49 \pm 1.72$ & 0.828 & $0.90 \pm 0.57$ & $1.60 \pm 1.78$ & 0.389 \\
\hline IL-4 & $1.56 \pm 1.31$ & $1.51 \pm 1.18$ & 0.877 & $1.38 \pm 1.17$ & $1.90 \pm 1.40$ & 0.139 \\
\hline MCP-1 & $38.96 \pm 75.08$ & $26.63 \pm 17.58$ & 0.354 & $38.34 \pm 80.34$ & $31.45 \pm 23.14$ & 0.097 \\
\hline IL-8 & $157.01 \pm 243.76$ & $122.74 \pm 211.38$ & 0.943 & $145.14 \pm 228.98$ & $156.15 \pm 252.77$ & 0.433 \\
\hline MIP-1A & $2.36 \pm 3.73$ & $3.04 \pm 2.39$ & 0.120 & $2.47 \pm 4.01$ & $2.59 \pm 2.12$ & 0.181 \\
\hline G-CSF & $118.82 \pm 73.74$ & $138.54 \pm 59.74$ & 0.174 & $109.84 \pm 66.38$ & $148.24 \pm 73.55$ & 0.028 \\
\hline IL-12p70 & $4.54 \pm 7.21$ & $5.11 \pm 8.68$ & 0.759 & $4.39 \pm 7.77$ & $5.18 \pm 7.70$ & 0.366 \\
\hline
\end{tabular}

Values are mean \pm standard deviation. FGF, fibroblast growth factor; G-CSF, granulocyte colony-stimulating factor; IL, interleukin; IP, interferon-inducible protein; MCP, monocyte chemotactic protein; MIP, macrophage inflammatory protein; PDGF, platelet-derived growth factor; SRAGE, soluble form of the receptor for advanced glycation end-products; TNF, tumor necrosis factor. 
Table S2 Demographics and risk factors for patients who had preoperative HLA samples tested for cytokine analysis using 2005 and 2016 guidelines

\begin{tabular}{|c|c|c|c|c|c|c|c|}
\hline \multirow{2}{*}{ Variable } & \multirow{2}{*}{ Total $(\mathrm{n}=60)$} & \multicolumn{3}{|c|}{2005 Guidelines } & \multicolumn{3}{|c|}{2016 Guidelines } \\
\hline & & Non-PGD $(n=46)$ & PGD $(n=14)$ & $P$ & Non-PGD ( $n=39)$ & PGD $(n=21)$ & $P$ \\
\hline \multicolumn{8}{|l|}{ Recipient characteristics } \\
\hline Female & $30(50.0)$ & 25 (54.35) & $5(35.71)$ & 0.360 & $21(53.85)$ & $9(42.86)$ & 0.589 \\
\hline Age (years) & $53.08 \pm 15.87$ & $52.65 \pm 15.93$ & $54.5 \pm 16.18$ & 0.706 & $52.1 \pm 16.7$ & $54.4 \pm 14.56$ & 0.519 \\
\hline $\mathrm{BMI}\left(\mathrm{kg} / \mathrm{m}^{2}\right)$ & $25.06 \pm 5.21$ & $24.21 \pm 5.08$ & $27.85 \pm 4.75$ & 0.021 & $23.46 \pm 4.95$ & $28.03 \pm 4.38$ & 0.0008 \\
\hline Blood type & & & 0.280 & & & & 0.222 \\
\hline$A$ & $24(40.0)$ & $16(34.78)$ & $8(57.14)$ & & $14(35.90)$ & $10(47.62)$ & \\
\hline B & $5(8.33)$ & $5(10.87)$ & $0(0.0)$ & & $5(12.82)$ & $0(0.0)$ & \\
\hline$A B$ & $3(5.0)$ & $3(6.52)$ & $0(0.0)$ & & $3(7.70)$ & $0(0.0)$ & \\
\hline $\mathrm{O}$ & $28(46.67)$ & $22(47.83)$ & $6(42.86)$ & & $17(43.59)$ & $11(52.38)$ & \\
\hline Rh group negative & $8(13.33)$ & $5(10.87)$ & $3(21.43)$ & 0.374 & $4(10.26)$ & $4(19.05)$ & 0.433 \\
\hline Primary disease & & & & 0.418 & & & 0.138 \\
\hline Restrictive lung disease & 32 (53.33) & $22(47.83)$ & $10(71.43)$ & & 17 (43.59) & $15(71.43)$ & \\
\hline Cystic fibrosis & $11(18.33)$ & $10(21.74)$ & $1(7.14)$ & & $10(25.64)$ & $1(4.76)$ & \\
\hline COPD & $13(21.67)$ & $11(23.91)$ & $2(14.29)$ & & $9(23.08)$ & $4(19.05)$ & \\
\hline Other & $4(6.67)$ & $3(6.52)$ & $1(7.14)$ & & $3(7.70)$ & $1(4.76)$ & \\
\hline Primary disease & & & & 0.140 & & & 0.058 \\
\hline Restrictive lung disease & $32(53.33)$ & $22(47.83)$ & $10(71.43)$ & & $17(43.59)$ & $15(71.43)$ & \\
\hline Non-restrictive lung diseases & $28(46.67)$ & $24(52.17)$ & $4(28.57)$ & & $22(56.41)$ & $6(28.57)$ & \\
\hline Diabetes & $10(16.67)$ & $8(17.39)$ & $2(14.29)$ & 1.000 & 7 (17.95) & $3(14.29)$ & 1.000 \\
\hline Hypertension & $26(43.33)$ & $20(43.48)$ & $6(42.86)$ & 1.000 & $16(41.03)$ & $10(47.62)$ & 0.785 \\
\hline History of smoking & $28(46.67)$ & $22(47.83)$ & $6(42.86)$ & 0.770 & $18(46.15)$ & $10(47.62)$ & 1.00 \\
\hline LAS & $42.24 \pm 12.26$ & $41.34 \pm 10.44$ & $45.22 \pm 17.10$ & 0.304 & $41.14 \pm 10.14$ & $44.29 \pm 15.53$ & 0.347 \\
\hline Baseline creatinine (mg/dL) & $0.83 \pm 0.19$ & $0.81 \pm 0.18$ & $0.87 \pm 0.23$ & 0.323 & $0.81 \pm 0.18$ & $0.85 \pm 0.21$ & 0.427 \\
\hline Condition at time of transplant & & & & 0.905 & & & 0.759 \\
\hline Not hospitalized & $52(86.67)$ & $40(86.96)$ & $12(85.71)$ & & $33(84.62)$ & $19(90.48)$ & \\
\hline Hospitalized (not in ICU) & $5(8.33)$ & $4(8.70)$ & $1(7.14)$ & & $4(10.26)$ & $1(4.76)$ & \\
\hline In ICU & $3(5.0)$ & $2(4.35)$ & $1(7.14)$ & & $2(5.13)$ & $1(4.76)$ & \\
\hline Life support before transplant & $4(6.67)$ & $2(4.35)$ & $2(14.29)$ & 0.230 & $2(5.13)$ & $2(9.52)$ & 0.606 \\
\hline Pre-operative ECMO & $2(3.33)$ & $1(2.17)$ & $1(7.14)$ & 0.415 & $1(2.56)$ & $1(4.76)$ & 1.00 \\
\hline Pulmonary hypertension & $49(81.67)$ & $38(82.61)$ & $11(78.57)$ & 0.708 & $32(82.05)$ & $17(80.95)$ & 1.00 \\
\hline Prior thoracic surgery & $10(16.67)$ & $7(15.22)$ & $3(21.43)$ & 0.685 & $4(10.26)$ & $6(28.57)$ & 0.143 \\
\hline Prior cardiac surgery & $1(1.67)$ & $1(2.17)$ & $0(0.0)$ & 1.000 & $1(2.56)$ & $0(0.0)$ & 1.00 \\
\hline Prior pleurodesis & $6(10.0)$ & $3(6.52)$ & $3(21.43)$ & 0.133 & $3(7.70)$ & $3(14.29)$ & 0.655 \\
\hline \multicolumn{8}{|l|}{ Donor characteristics } \\
\hline Age (years) & $33.6 \pm 12.73$ & $33.87 \pm 12.73$ & $32.71 \pm 13.89$ & 0.769 & $33.15 \pm 12.87$ & $34.43 \pm 12.71$ & 0.714 \\
\hline Extended criteria donor† & $19(31.67)$ & $12(26.09)$ & $7(50.00)$ & 0.092 & $11(28.21)$ & $8(38.10)$ & 0.432 \\
\hline Smoker ever & $31(51.67)$ & $24(52.17)$ & $7(50.0)$ & 1.000 & $21(53.85)$ & $10(47.62)$ & 0.788 \\
\hline Smoker $>20 \mathrm{PYH}$ & $5(8.33)$ & $4(8.70)$ & $1(7.14)$ & 1.000 & $4(10.26)$ & $1(4.76)$ & 0.649 \\
\hline DCD & $5(8.33)$ & $3(6.52)$ & $2(14.29)$ & 0.582 & $2(5.13)$ & $3(14.29)$ & 0.332 \\
\hline \multicolumn{8}{|l|}{ Operative characteristics } \\
\hline Bilateral type of transplant & $60(100.0)$ & $46(100.0)$ & $14(100.0)$ & & $39(100.0)$ & $21(100.0)$ & \\
\hline Type of intraoperative support & & & & 1.000 & & & 1.000 \\
\hline Off-pump & $1(1.67)$ & $1(2.17)$ & $0(0.0)$ & & $1(2.56)$ & $0(0.0)$ & \\
\hline ECLS & 59 (98.33) & $45(97.83)$ & $14(100.0)$ & & 38 (97.44) & $21(100.0)$ & \\
\hline Type of ECLS & & & & 1.000 & & & 1.000 \\
\hline ECMO & $4(6.67)$ & $3(6.67)$ & $1(7.14)$ & & $3(7.89)$ & $1(4.76)$ & \\
\hline CPB & 55 (93.22) & 42 (93.33) & $13(92.86)$ & & 35 (92.11) & 20 (95.24) & \\
\hline Total ischemic time (min) & $295.77 \pm 63.59$ & $290.15 \pm 66.33$ & $314.2 \pm 51.41$ & 0.218 & $296.15 \pm 65.34$ & $295.05 \pm 61.77$ & 0.949 \\
\hline
\end{tabular}

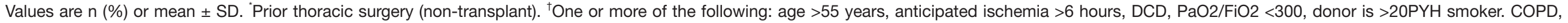

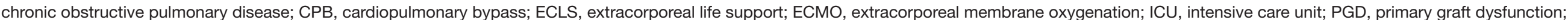
$\mathrm{PYH}$, pack-year history; CPB, cardiopulmonary bypass; DCD, donor after cardiac death; LAS, lung allocation score. 
Table S3 Clinical characteristics, outcomes, and biomarkers in patients with PGD according to 2005 and 2016 guidelines and in those downgraded to PGD1 using the 2005 guidelines

\begin{tabular}{|c|c|c|c|}
\hline Variable & PGD 2016 \& $2005(n=33)$ & PGD 2016 not $2005(n=14)$ & $\mathrm{P}$ \\
\hline \multicolumn{4}{|l|}{ Recipient characteristics } \\
\hline Women & $10(30.30)$ & $6(42.86)$ & 0.406 \\
\hline Age & $54.45 \pm 15.85$ & $55.50 \pm 12.81$ & 0.828 \\
\hline $\mathrm{BMI}\left(\mathrm{kg} / \mathrm{m}^{2}\right)$ & $26.15 \pm 5.15$ & $27.41 \pm 4.13$ & 0.423 \\
\hline Primary disease & & & 0.243 \\
\hline Restrictive lung disease & $24(72.72)$ & $7(50.50)$ & \\
\hline Non-restrictive lung diseases & $9(27.28)$ & $7(50.50)$ & \\
\hline Diabetes & $11(33.33)$ & $4(28.57)$ & 1.00 \\
\hline Hypertension & $17(51.52)$ & $9(64.29)$ & 0.421 \\
\hline History of smoking & $16(48.48)$ & $9(64.29)$ & 0.321 \\
\hline Lung allocation score & $45.35 \pm 14.17$ & $41.03 \pm 9.43$ & 0.302 \\
\hline Baseline creatinine $(\mathrm{mg} / \mathrm{dL})$ & $0.85 \pm 0.20$ & $0.89 \pm 0.21$ & 0.639 \\
\hline Condition at time of transplant & & & 0.582 \\
\hline Not hospitalized & $28(84.85)$ & $14(100.0)$ & \\
\hline Hospitalized (not in ICU) & $2(6.06)$ & $0(0)$ & \\
\hline In ICU & $3(9.09)$ & $0(0)$ & \\
\hline Life support before LTx & $4(12.12)$ & $0(0)$ & 0.302 \\
\hline Pre-operative ECMO & $1(3.03)$ & $0(0)$ & 0.7 \\
\hline Pulmonary hypertension & $23(69.70)$ & $13(92.86)$ & 0.181 \\
\hline Prior thoracic surgery (non-transplant) & $6(18.18)$ & $3(21.43)$ & 1.00 \\
\hline Prior cardiac surgery & $0(0)$ & $1(7.14)$ & 0.298 \\
\hline Prior pleurodesis & $3(9.09)$ & $0(0)$ & 0.544 \\
\hline Prior lung transplant & $1(3.03)$ & $1(7.14)$ & 0.879 \\
\hline \multicolumn{4}{|l|}{ Donor characteristics } \\
\hline Age (years) & $33.67 \pm 12.88$ & $38.21 \pm 10.39$ & 0.249 \\
\hline Extended criteria donor & $16(48.48)$ & $4(28.57)$ & 0.334 \\
\hline Smoker ever & $19(57.58)$ & $7(50.00)$ & 0.633 \\
\hline Sex mismatch & $12(36.36)$ & $5(35.71)$ & 0.772 \\
\hline Sex mismatch, female-male & $9(27.28)$ & $2(14.29)$ & 0.559 \\
\hline Donor after cardiac death & $6(18.18)$ & $1(7.14)$ & 0.657 \\
\hline Ex-vivo lung perfusion & $3(9.09)$ & $1(7.14)$ & 1.00 \\
\hline \multicolumn{4}{|l|}{ Operative characteristics } \\
\hline Type of transplant & & & 0.627 \\
\hline Bilateral & $30(90.91)$ & $12(85.71)$ & \\
\hline Single & $3(9.09)$ & $2(14.29)$ & \\
\hline Type of intraoperative support & & & 0.298 \\
\hline Off-pump & $0(0)$ & $1(7.14)$ & \\
\hline ECLS & $33(100.0)$ & $13(92.86)$ & \\
\hline Type of ECLS & & & 0.849 \\
\hline Cardiopulmonary bypass & $28(84.85)$ & $12(92.31)$ & \\
\hline Modified bypass & $5(15.15)$ & $1(7.69)$ & \\
\hline Total ischemic time (min) & $346.30 \pm 118.45$ & $301.5 \pm 128.44$ & 0.253 \\
\hline \multicolumn{4}{|l|}{ Outcome } \\
\hline Postoperative length of stay (days) & $46.30 \pm 40.97$ & $20.93 \pm 13.47$ & 0.029 \\
\hline ICU length of stay (days) & $37.33 \pm 36.07$ & $11.71 \pm 13.33$ & 0.135 \\
\hline Peak creatinine within 48 hours $(\mathrm{mg} / \mathrm{dL}$ ) & $1.23 \pm 0.59$ & $0.93 \pm 0.19$ & 0.073 \\
\hline Peak creatinine value $<1.5 \mathrm{mg} / \mathrm{dL}$ & $25(75.76)$ & $14(100.0)$ & 0.084 \\
\hline Peak creatinine value $\geq 1.5 \mathrm{mg} / \mathrm{dL}$ & $8(24.24)$ & $0(0)$ & 0.084 \\
\hline Max creatinine (mg/dL) & $1.96 \pm 1.40$ & $1.48 \pm 0.85$ & 0.260 \\
\hline Max creatinine $<1.5 \mathrm{mg} / \mathrm{dL}$ & $15(45.45)$ & $11(78.57)$ & 0.055 \\
\hline Max creatinine $\geq 1.5 \mathrm{mg} / \mathrm{dL}$ & $18(54.55)$ & $3(21.43)$ & 0.055 \\
\hline Discharge creatinine $(\mathrm{mg} / \mathrm{dL})$ & $1.05 \pm 1.05$ & $0.98 \pm 0.89$ & 0.420 \\
\hline $\begin{array}{l}\text { Peak lactate within } 72 \text { hours }(\mathrm{mg} / \mathrm{dL}) \\
(\mathrm{n}=115)\end{array}$ & $9.02 \pm 3.64$ & $5.49 \pm 3.16$ & 0.004 \\
\hline Postoperative ECMO & $15(45.45)$ & $0(0)$ & 0.002 \\
\hline Mechanical ventilation $\geq 48$ hours & $29(87.88)$ & $3(21.43)$ & 0.000 \\
\hline Mechanical ventilation $\geq 5$ days & $22(66.67)$ & $1(7.14)$ & 0.000 \\
\hline Reintubated & $11(33.33)$ & 2 (14.29) & 0.288 \\
\hline Tracheostomy & $18(54.55)$ & $1(7.14)$ & 0.003 \\
\hline Airway dehiscence & $3(9.09)$ & $0(0)$ & 0.544 \\
\hline Dialysis & $12(36.36)$ & $0(0)$ & 0.009 \\
\hline Discharge disposition & & & 0.164 \\
\hline Home & $18(54.55)$ & $11(78.57)$ & \\
\hline Rehabilitation facility & $8(24.24)$ & $3(21.43)$ & \\
\hline Death & $7(21.21)$ & $0(0)$ & \\
\hline 90-day mortality & $4(12.12)$ & $0(0)$ & 0.302 \\
\hline 1-year mortality & $11(33.33)$ & $1(7.14)$ & 0.077 \\
\hline \multicolumn{4}{|l|}{ Biomarkers } \\
\hline IL-2 ( $n=11$ vs. 5) & $6.65 \pm 6.45 \mathrm{pg} / \mathrm{mL}$ & $3.30 \pm 1.06 \mathrm{pg} / \mathrm{mL}$ & 0.276 \\
\hline 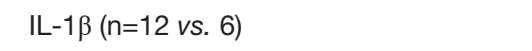 & $0.59 \pm 0.55 \mathrm{pg} / \mathrm{mL}$ & $0.69 \pm 0.88 \mathrm{pg} / \mathrm{mL}$ & 0.771 \\
\hline PDGF-BB ( $n=14$ vs. 7 ) & $1834.37 \pm 1524.54 \mathrm{pg} / \mathrm{mL}$ & $2384.50 \pm 1740.78 \mathrm{pg} / \mathrm{mL}$ & 0.457 \\
\hline G-CSF ( $n=14$ vs. 7 ) & $211.20 \pm 377.85 \mathrm{pg} / \mathrm{mL}$ & $166.25 \pm 97.02 \mathrm{pg} / \mathrm{mL}$ & 0.685 \\
\hline SRAGE ( $n=14$ vs. 7 ) & $807.87 \pm 544.27 \mathrm{pg} / \mathrm{mL}$ & $726.56 \pm 329.39 \mathrm{pg} / \mathrm{mL}$ & 0.722 \\
\hline
\end{tabular}

Values are mean \pm standard deviation. PGD 2016 \& 2005, patients who developed PGD3 at 48-72 hours after transplant using the 2016 and 2005 scoring guidelines; PGD 2016 not 2005, patients who developed PGD3 at 48-72 hours after lung transplant using the 2016 guidelines but not when using the 2005 guidelines. IL, interleukin; PDGF, platelet-derived growth factor; GCSF, granulocyte colony 
Table S4 Outcomes for 60 patients who had preoperative HLA samples tested for cytokine analysis using 2005 and 2016 guidelines

\begin{tabular}{|c|c|c|c|c|c|c|c|}
\hline \multirow{2}{*}{ Variable } & \multirow{2}{*}{ Total $(n=60)$} & \multicolumn{3}{|c|}{2005 Guidelines } & \multicolumn{3}{|c|}{2016 Guidelines } \\
\hline & & Non-PGD $(n=46)$ & PGD $(n=14)$ & $P$ & Non-PGD ( $n=39)$ & $P G D(n=21)$ & $P$ \\
\hline Postoperative length of stay (days) & & & & 0.047 & & & 0.178 \\
\hline$\leq 21$ & $31(51.67)$ & $26(56.52)$ & $5(35.71)$ & & $21(53.85)$ & $10(47.62)$ & \\
\hline $22-30$ & $14(23.33)$ & $12(26.09)$ & $2(14.29)$ & & $11(28.21)$ & $3(14.29)$ & \\
\hline$>30$ & $15(25.00)$ & $8(17.39)$ & $7(50.0)$ & & $7(17.95)$ & $8(38.10)$ & \\
\hline ICU length of stay (days) & $18.85 \pm 27.24$ & $15.06 \pm 27.24$ & $31.29 \pm 35.27$ & 0.050 & $15.1 \pm 24.55$ & $25.81 \pm 31.07$ & 0.148 \\
\hline Peak creatinine within 48 hours (mg/dL) & $1.05 \pm 0.48$ & $1.00 \pm 0.48$ & $1.22 \pm 0.45$ & 0.140 & $1.02 \pm 0.51$ & $1.11 \pm 0.41$ & 0.526 \\
\hline Peak creatinine within 48 hours $\geq 1.5(\mathrm{mg} / \mathrm{dL})$ & $6(10.0)$ & $2(4.35)$ & $4(28.57)$ & 0.023 & $2(5.13)$ & $4(19.05)$ & 0.171 \\
\hline Max creatinine (mg/dL) & $1.46 \pm 1.16$ & $1.43 \pm 1.16$ & $1.56 \pm 0.83$ & 0.710 & $1.47 \pm 1.31$ & $1.47 \pm 0.84$ & 0.954 \\
\hline Max creatinine $\geq 1.5(\mathrm{mg} / \mathrm{dL})$ & $17(28.33)$ & $10(21.74)$ & $7(50.0)$ & 0.040 & $9(23.08)$ & $8(38.10)$ & 0.176 \\
\hline Discharge creatinine (mg/dL) & $0.87 \pm 0.51$ & $0.87 \pm 0.51$ & $0.86 \pm 0.37$ & 0.930 & $0.91 \pm 0.59$ & $0.84 \pm 0.39$ & 0.479 \\
\hline Peak lactate within $72 \mathrm{~h}(\mathrm{mg} / \mathrm{dL})(\mathrm{n}=55)$ & $7.12 \pm 3.51$ & $6.54 \pm 3.51$ & $8.81 \pm 3.04$ & 0.036 & $6.62 \pm 3.59$ & $7.99 \pm 3.27$ & 0.164 \\
\hline Postoperative ECMO & $4(6.64)$ & $1(2.17)$ & $3(21.43)$ & 0.036 & $1(2.56)$ & $3(14.29)$ & 0.119 \\
\hline Mechanical ventilation $\geq 48 \mathrm{~h}$ & $28(46.67)$ & $16(34.78)$ & $12(85.71)$ & 0.002 & $14(35.90)$ & $14(66.67)$ & 0.031 \\
\hline Mechanical ventilation $\geq 5$ days & $18(30.0)$ & $9(19.57)$ & $9(64.29)$ & 0.003 & $8(20.51)$ & $10(47.62)$ & 0.040 \\
\hline Reintubated & $19(31.67)$ & $15(32.61)$ & $4(28.57)$ & 1.000 & $14(35.90)$ & $5(23.81)$ & 0.254 \\
\hline Tracheostomy & $15(25.0)$ & $9(19.57)$ & $6(42.86)$ & 0.092 & $8(20.51)$ & 7 (33.33) & 0.352 \\
\hline Airway dehiscence & $1(1.67)$ & $0(0.0)$ & $1(7.14)$ & 0.233 & $0(0.0)$ & $1(4.76)$ & 0.350 \\
\hline Dialysis & $6(10.0)$ & $4(8.70)$ & $2(14.29)$ & 0.617 & $4(10.26)$ & $2(9.52)$ & 1.00 \\
\hline Discharge disposition & & & & 0.312 & & & 0.1743 \\
\hline Home & $46(76.67)$ & $37(80.43)$ & $9(64.29)$ & & $33(84.62)$ & $13(61.90)$ & \\
\hline Rehabilitation facility & $13(21.67)$ & $8(17.39)$ & $5(35.71)$ & & $6(15.38)$ & 7 (33.33) & \\
\hline Death & $1(1.67)$ & $1(2.17)$ & $0(0.0)$ & & $1(2.56)$ & $0(0.0)$ & \\
\hline Readmission within 1 year $(n=59)$ & $50(84.75)$ & $39(86.67)$ & $11(78.57)$ & 0.431 & $33(86.84) n=38$ & 17 (80.95) & 0.708 \\
\hline ICU readmission within 1 year $(n=59)$ & $20(33.9)$ & $14(31.11)$ & $6(42.86)$ & 0.521 & $11(28.95) n=38$ & $9(42.86)$ & 0.390 \\
\hline 90-day mortality & $0(0.0)$ & $0(0.0)$ & $0(0.0)$ & & $0(0.0)$ & $0(0.0)$ & \\
\hline 1-year mortality & $4(6.64)$ & $3(6.52)$ & $1(7.14)$ & 1.000 & $2(5.13)$ & $2(9.52)$ & 0.606 \\
\hline
\end{tabular}

Values are $\mathrm{n}(\%)$ or mean $\pm \mathrm{SD}$. ECMO, extracorporeal membrane oxygenation; ICU, intensive care unit. 